\title{
From bore-soliton-splash to rogue waves, a new wave-energy device and extreme tsunami run-up
}

\author{
O. Bokhove ${ }^{1}$, A. Kalogirou ${ }^{2}$, W. Zweers ${ }^{3}$ \\ ${ }^{1}$ School of Mathematics, University of Leeds, Leeds, LS2 9JT, UK \\ ${ }^{2}$ School of Mathematics, University of East Anglia, Norwich Research Park, Norwich \\ NR4 7TJ, UK \\ ${ }^{3}$ WowLab, Roombeek, Stroinksbleekweg 4a, Enschede, 7523 ZL, The Netherlands \\ E-mail: o.bokhove@leeds.ac.uk, zweers@dds.nl, anna.kalogirou@uea.ac.uk
}

\begin{abstract}
We explore extreme nonlinear water-wave amplification in a contraction or, analogously, wave amplification in crossing seas. The latter case can lead to extreme or rogue-wave formation at sea. First, amplification of a solitary-water-wave compound running into a contraction is disseminated experimentally, for small-scale and larger wavetanks. Maximum amplification in our bore-soliton-splash observed is circa tenfold. Subsequently, nonlinear and numerical modelling approaches are developed and validated for amplifying, contracting waves. Amplification phenomena observed have led us to develop a novel wave-energy device with wave amplification in a contraction used to enhance wave-buoy motion and magnetically-induced energy generation. An experimental proof-of-principle shows that our wave-energy device works. Furthermore, we develop a monolithic, mathematical model of wave hydrodynamics, buoy motion and electric power generation by magnetic induction, satisfying one grand variational principle in its conservative limit. Dissipative features, electrical wire resistance and nonlinear LED-loads, are added a posteriori. Preliminary simulations of our simplified (linear) wave-energy model are encouraging. Further highlights discussed are: exact modelling of crossing seas with KadomtsevPetviashvili's equation, bore-soliton-splash' relevance to devastating Tohoku tsunami run-up in 2011, nonlinear wave-energy optimisation and a steel-soliton-splash artwork. $\ddagger$
\end{abstract}

\section{Introduction}

Early September 2010, three applied mathematicians at the University of Twente made requests to create a soliton in a make-shift wavetank for a new "research plaza" opening festivity. Part of that plaza contains a water feature or channel approximately $45 \mathrm{~m}$ long, $2 \mathrm{~m}$ wide and $1.2 \mathrm{~m}$ deep. Normally filled to its edge with water and harbouring water plants and fish, at the time it was only partially filled. In August 1834, John Scott Russell discovered a soliton while riding his horse along the Edinburgh Union Canal. This single "wave of elevation", as Scott Russell called it, caused by sudden

$\ddagger$ Note that this is a non-peer reviewed preprint submitted to EarthArXiv. 

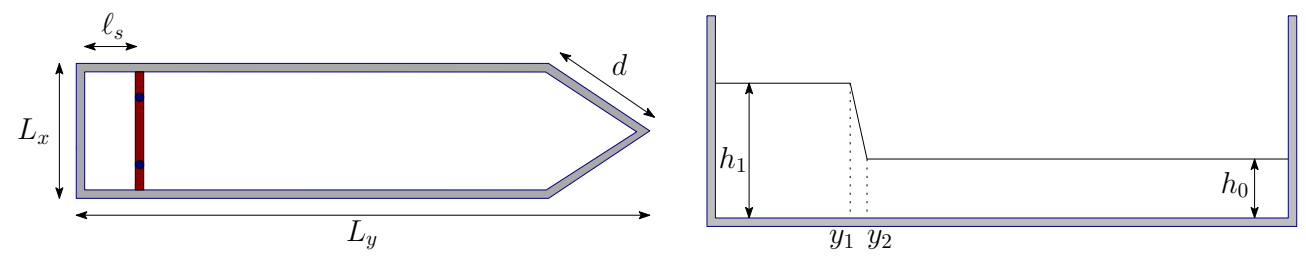

Figure 1. Wave-channel set-up sketch with top and side views (left/right). Sluice-gate speed determined approximately by video analysis of the sluice-gate removal.

Table 1. Details of soliton-splash experiment, including wavetank dimensions.

\begin{tabular}{ll}
\hline Wavetank length & $L_{y}=43.63 \pm 0.1 \mathrm{~m}$ \\
Wavetank width & $L_{x}=2 \mathrm{~m}$ \\
Wavetank height & $L_{z}=1.2 \mathrm{~m}$ \\
Contraction length & $d=2.7 \mathrm{~m}$ \\
Location of sluice gate & $\ell_{s}=2.63 \mathrm{~m}$ \\
Rest-water level (high) & $h_{1}=0.9 \mathrm{~m}$ \\
Rest-water level (low) & $h_{0}=0.43 \mathrm{~m}$ \\
Sluice-gate release speed & $V_{g} \approx 2.5 \mathrm{~m} / \mathrm{s}$ \\
Sluice-gate removal time & $T_{s}=h_{1} / V_{g} \approx 0.36 \mathrm{~s}$ \\
\hline
\end{tabular}

halting of a narrow boat, travelled several miles as spatially coherent heap of water, at a nearly constant speed, before wave height gradually diminished after two miles. In 1895, Korteweg and De Vries derived the exact sech-wave solution for the so-called Kortewegde-Vries equation [32], derived as an approximation to potential-flow equations for incompressible water waves. A soliton is a wave with nonlinearity and dispersion in balance such that the wave stays coherent and neither disperses nor breaks [13]. Scott Russell created solitons in a 30-feet laboratory channel. Solitons or solitary waves can be generated at the beginning of a rectangular channel with vertical walls: by using either a piston moving bespokely, a block lowered at a finite yet fast speed into the water or by a quick sluice-gate removal between a higher rest-water level $\left(h_{1}\right)$ lock section and a lower rest-water level $\left(h_{0}\right)$ main section. We have used the latter for solitary-wave generation with an extra channel feature, sketched in Fig. 1 with dimensions in Table 1: a V-shaped channel end with vertical walls.

While a soliton is a well-known mathematical and fluid-mechanical feature in nonlinear science, a general audience would be entertained more when such a travelling heap of water would lead to an extreme wave, to mark the plaza opening. We therefore added the $\mathrm{V}$-shaped contraction to create the highest splash possible by only varying water levels $h_{0}$ and $h_{1}$ before and after the sluice gate, given a wavechannel geometry. Ideas for wave height amplification arose from work on hydraulic flow stowage in contractions [2] and on wave impact against sea walls by Peregrine [46, 7], 


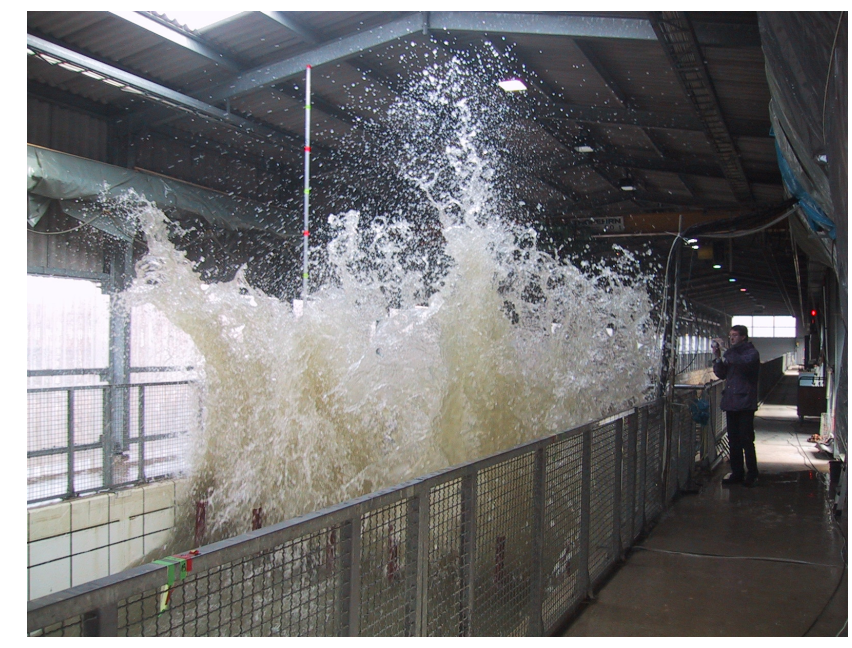

Figure 2. Wave impact against a wall in hydraulic facilities in Hannover, Germany. Photo courtesy: D. Howell Peregrine's slide inherited by O.B. via the School of Mathematics, University of Bristol.

cf. Fig. 2. After some trials in two wavetanks, we created a "bore-soliton-splash" $§$. The highest bore-soliton-splash -Case 8 in Table 2- consisted of time-dependent evolution summarised in Fig. 3: a) water initially at rest with b) an excavator ready to lift the sluice gate out of the channel; after sluice gate removal a coherent compound of "2.5" solitary waves travels towards the contraction and the highest solitary wave breaks shortly after its inception into c) a so-called hydraulic bore or spilling breaker; the slightly lower non-breaking second wave is tailed by a lower third wave, with all three waves travelling at a slightly different speeds; during its propagation, the bore diminishes in height due to turbulent energy dissipation and d) breaking ceases just before the contraction; the smoothened wave then amplifies and reflects in the V-shaped channel contraction and upon reflection draws a deep trough in which the second slightly lower wave crashes precisely, leading to e,f) generation of a wave or splash circa ten times the incoming, first and highest solitary wave height. The bore-soliton-splash let to a variety of new ideas including its modelling and the creation and testing of a proof-of-concept of an inspired wave-energy device. Our paper aims to highlight and partially investigate these ideas to relate this splash or man-made rogue wave to similar rogue or monster waves in our oceans.

Rogue, monster or extreme waves are anomalously high and rare waves with wave height $H_{r w}$, generally considered at sea, defined relative to a significant or ambient-wave height $H_{s}$ of surrounding, preceding and following seas. A straightforward definition of rogue waves states that the abnormality index $A I=H_{r w} / H_{s}>2$, i.e. the rogue-wave height $H_{r w}$ must be at least twice as high as the ambient-wave height $H_{s}$. Dysthe et al. [14] and Khariff et al. [30] provide more advanced and precise $\S$ Designer and artist WZ named the "soliton splash" 20-09-2010 video with "bore" indicating intermittent wave breaking. 
a)

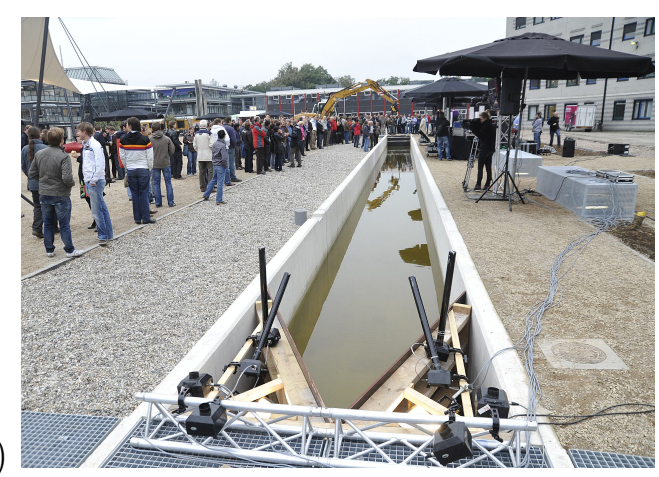

b)
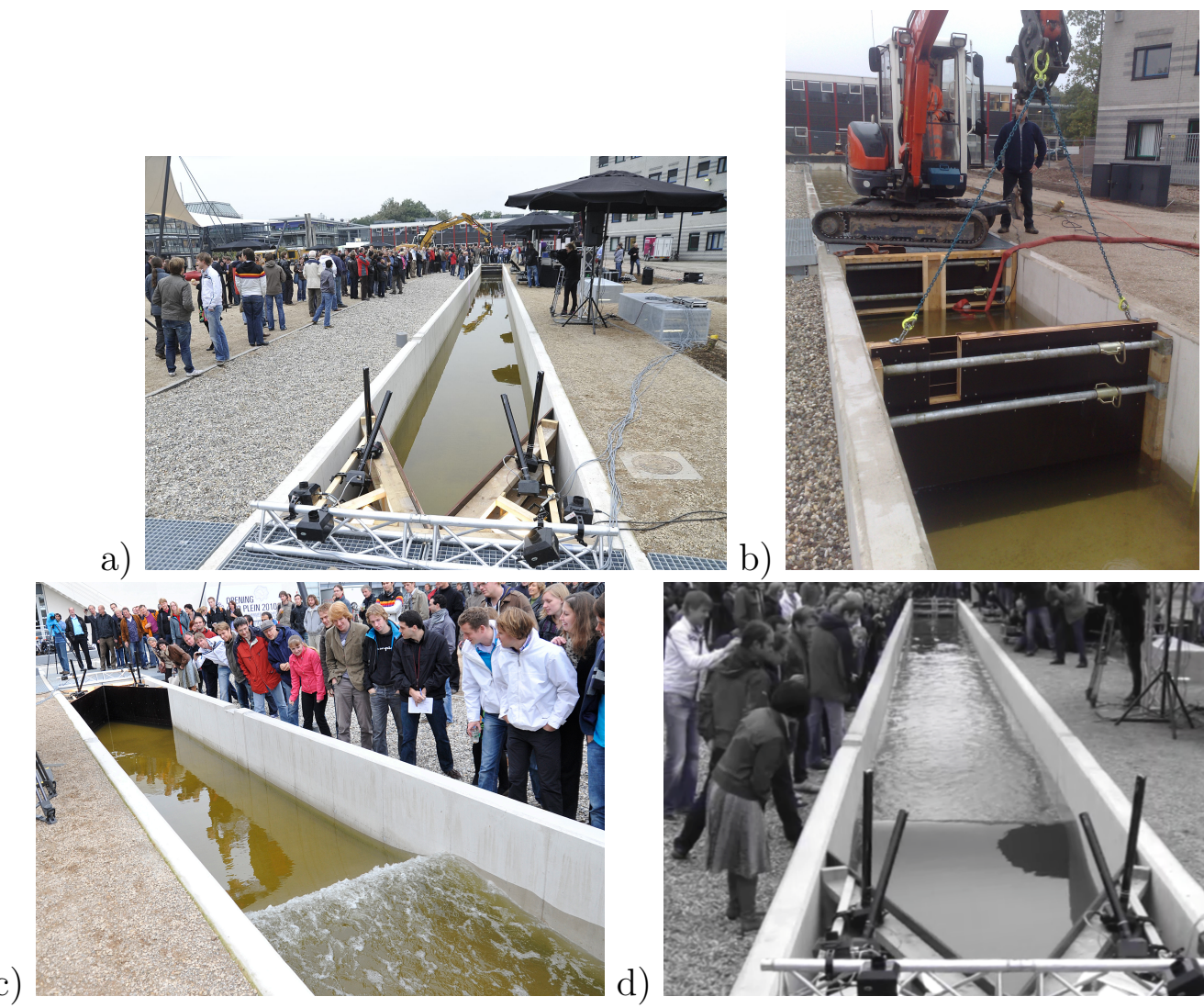

d)

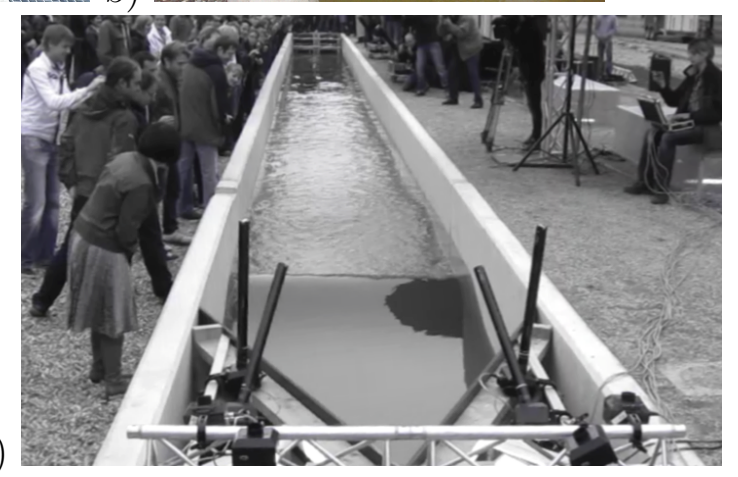

e)

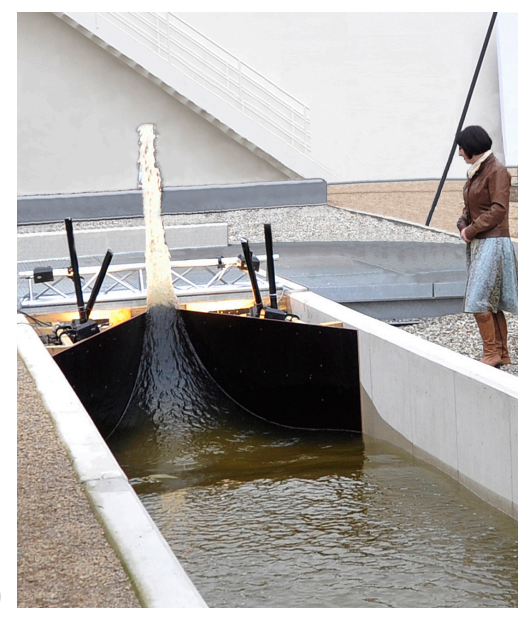

f)

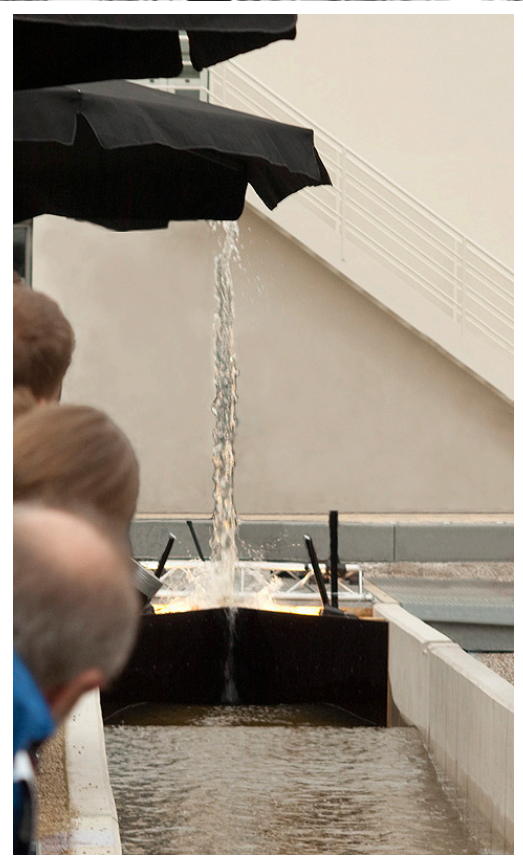

Figure 3. Time evolution snapshots of the highest bore-soliton-splash, Case 8 in Table 2. a) Channel overview before sluice gate is removed. b) Sluice gate with excavator used to smoothly remove the sluice gate. After sluice gate removal c) the highest solitary wave in the compound becomes a bore or spilling breaker, dissipating turbulent energy and diminishing amplitude while it propagates to become d) smooth again before the contraction. After reflection it draws a trough in which the second wave falls thus forming e) a jet f) collapsing after reaching its apex. 
definitions of rogue waves, but this common definition above suffices here. Rogue waves have a rare, extreme occurrence and are therefore difficult to predict, either statistically or deterministically. Understanding their wave height and occurrence is relevant to maritime and coastal engineering given their potential to damage ships, maritime and coastal structures, including sinking and disappearance of ships; an overview of such disasters is found in Nikolkina and Didulenkova [41]. There are different types of rogue waves, involving linear and nonlinear wave focussing in one horizontal dimension, spatial wave focussing due to coastal or submarine convergences, episodic waves such as tsunamis generated elsewhere, and crossing seas with pyramidal waves $[8,16]$. These different rogue-wave types have been (partially) explained within a hierarchy of different models, including, e.g., incompressible Euler equations with a free surface and passive or limited air motion, its potential-flow restriction, and numerous asymptotic approximations of these classical potential-flow water-wave equations such as Benney-Luke equations, Kadomtsev-Petviasvili's (KP) equation, nonlinear Schrodinger equation(s) and the Korteweg-De-Vries equation [30, 13, 43, 27, 42, 39].

The main results achieved in this article are partially conceptual including:

- a detailed description of a man-made bore-soliton splash rogue-wave with an abnormality index of $A I=10$.

- establishing and employing mathematical and numerical models for experimental Cases 8 and 9 (see Table 2); with improved simulations beyond the one in Bokhove and Kalogirou [5].

- Inspired by the bore-soliton-splash configuration, we invented a novel rogue-waveenergy device, and built and tested a scaled-down version; a first nonlinear mathematical model is developed here, for which we show preliminary linear simulations.

- Albeit with (complicated) exact solutions of the simplified KP equation, we model the soliton-splash amplification to some extent exactly and relate it to rogue waves in crossing open-ocean seas as opposed to our closed-channel rogue waves.

- We discuss how the bore-soliton-splash relates to extreme 2011 Tohoku tsunami run-up in Japanese coastal estuaries and valleys.

The outline of our paper is as follows. Soliton-splash and bore-soliton-splash experiments are analysed in $\S 2$. Mathematical and numerical solutions of various models are developed and shown in $\S 3$, including simulations of Benney-Luke's model in §3.1. In $\S 4$ the wave-energy device is introduced; with one comprehensive and novel, nonlinear mathematical model of hydrodynamics, buoy motion and power generation; and, after using a novel compatible discretisation of that model, the first numerical modelling results are presented for its linearised dynamics. We finish with a discussion of open questions and challenges in $\S 5$, also highlighting exact solutions of the KP equation regarding an idealised splash, extreme rogue-wave run-up of the Tohoku 2011 tsunami as well as an splash-inspired artwork. 


\section{Experimental set-up and results}

Our goal was to create both a travelling soliton by removing a sluice gate separating two different water levels, initially at rest, and a splash of the highest possible amplitude in a V-shaped contraction. Given time constraints, the only way to determine whether our goal was reachable in practice was to resort to experimentation in two make-shift wave channels: where Roombeek the brook flows onto the University of Twente campus, see Fig. 4 (half of a V-shaped contraction was used), and on above-mentioned Research Plaza, see Fig. 3. This "optimisation" process of highest splash creation was constrained by channel geometry, with wave-contraction angle and channel length fixed a priori, while initial water levels $h_{0}$ and $h_{1}$ were varied to some extent. To create a solitary wave or soliton it is important to have intermediate water depth, as opposed to deep or shallow water. For the Roombeek channel we used water levels $h_{1}=0.57 \pm 0.01 \mathrm{~m}$ and $h_{0} \in[0.26,0.30] \pm 0.01 \mathrm{~m}$ and for the Plaza channel we used $h_{0} \in[0.32,0.47] \pm 0.01 \mathrm{~m}$ and $h_{1} \in[0.67,1.02] \pm 0.01 \mathrm{~m}$. In the smaller Roombeek channel the slanted contraction wall was required to have strong support, see Fig. 4a,c), against surprisingly high waveimpact forces, see Fig. 4d). On 20-09-2010, we managed to obtain two soliton splashes with $h_{1} \approx 2 h_{0}$, cf. Fig. 4 , convincing us it was possible to make a larger and reproducible Plaza-channel soliton-splash. A V-shaped channel end was constructed, see Fig. 3a), to withstand strong wave-impact forces and the sluice gate was constructed, see Fig. 3b), to withstand both hydraulic pressure and impose minimal obstruction to water flow, via small wooden strips on walls and bottom, as seen in Fig. 3b), once lifted.

Seven test cases were completed on 27-09-2010, including six with different restwater levels $h_{0}$ and $h_{1}$, and one repeated case with the highest splash to ensure reproducibility on the opening day of the Research Plaza. The optimal case involved $h_{0}=0.41 \pm 0.01 \mathrm{~m}$ and $h_{1}=0.9 \pm 0.01 \mathrm{~m}$. These two repeat cases and the general outcomes on 27-09-2010 showed that our experiments to create a bore-soliton-splash were reproducible on the opening day (30-09-2010). All cases are summarised and dated in Table 2 with repeat cases underlined and numbered by 3, 6 and 8. On 30-09-2010, this "optimal" case was successfully repeated, as shown in Fig. 3, followed by a case numbered 9 with the higher water level of $h_{0}=0.43 \mathrm{~m}$ set in the main channel by the addition of sluice gate's extra water from optimal Case 8 , while keeping $h_{1}=0.9 \mathrm{~m}$; Case 9 resulted in a smooth solitary-wave compound without wave breaking and a lower splash. Its evolution in time is displayed in Fig. 5 alongside bespoke simulations introduced and explained later. Videos of (nearly) all cases are found on Zweers' YouTube channel [48] and numbered accordingly. Inspection of videos of three repeat Cases 3, 6 and 8 reveals that there are some/minor differences, partially commented on in Table 2 . We attribute differences to the estimated error of $\pm 0.01 \mathrm{~m}$ in initial water levels $h_{0,1}$ and the manner of sluice gate removal by the excavator, despite training to be as consistent as possible. Case 9 underscores these sensitivities to initial conditions because a $0.02 \mathrm{~m}$ change from $h_{0}=0.41 \mathrm{~m}$ (Cases 3,6 and 8) to $0.43 \mathrm{~m}$, while keeping $h_{1}=0.9 \mathrm{~m}$, within measurement error, led to a quite different splash. Note, however, that the outcomes are not chaotic, 
a)

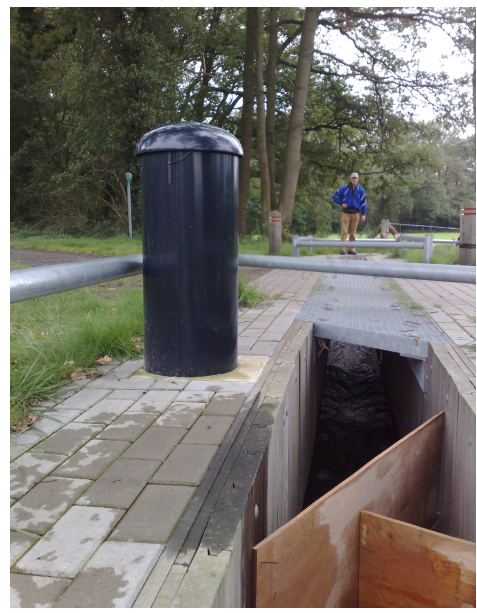

c)

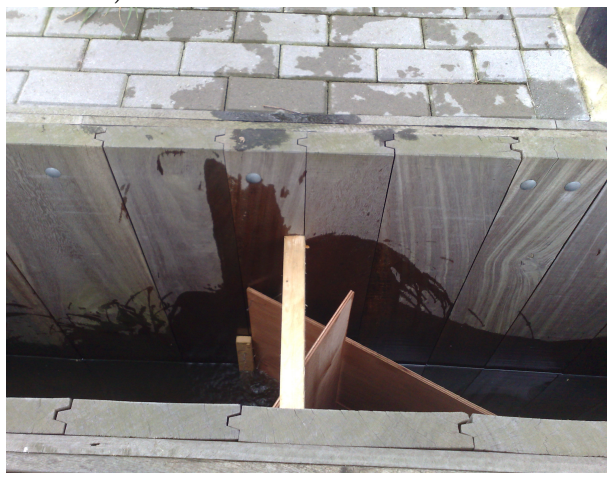

b)

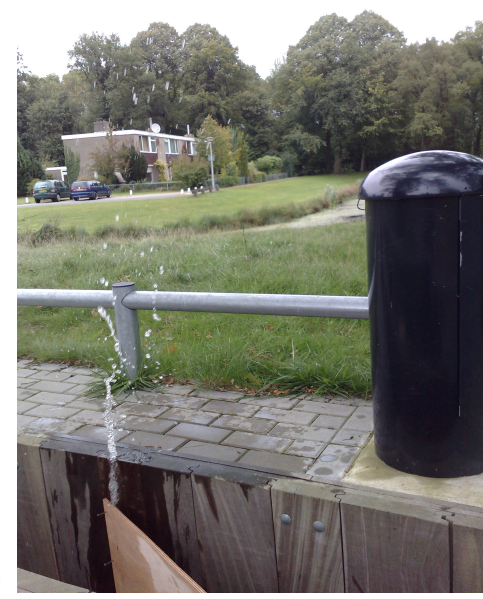

d)

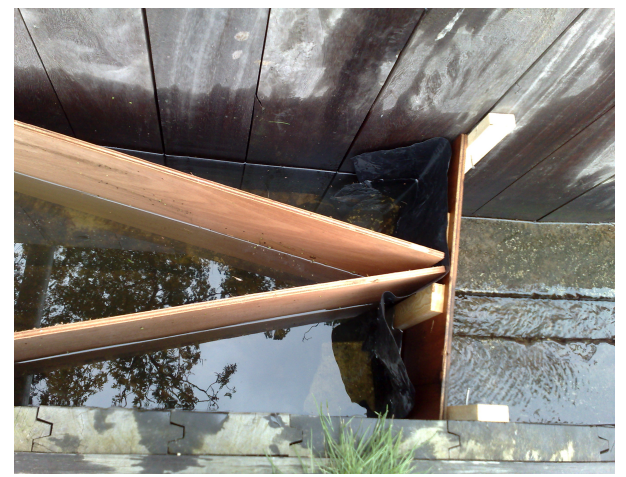

Figure 4. Small-scale pilot tests undertaken (Sunday 20-09-2010) in the "Roombeek" channel, a small brook with relatively constant water influx, at the edge of University of Twente's campus. a) Overview of channel with the foreground slanted channel end, fixed securely against wave impact with channel start and sluice gate where O.B. is standing. c) Contraction with water wall markings and b) collapsing splash at contraction apex. d) The first, V-shaped contraction was blown apart by wave impact due to insufficient, self-sustaining support on its downstream side. A video link of two splashes is found in [48].

as three reasonably repeatable cases demonstrate.

Several splash types were observed in the nine cases including minor/major reflections, resonances between waves (Cases 3,6 and 8), smooth waves, sheets (Case 9) and pyramidal waves (Cases 3, 6 and 8). Rogue-wave amplitudes found ranged between $H_{r w} \in[0.6,3.5] \pm 0.5 \mathrm{~m}$. For the highest cases of the bore-soliton-splash we found $H_{s}=0.35 \pm 0.05 \mathrm{~m}$ for the highest and first solitary wave and a rogue-wave height of $H_{r w}=[3.25,3.5,3.5] \pm 0.5 \mathrm{~m}$ leading to a maximum abnormality index of

$$
A I=\frac{H_{r w}}{H_{s}} \in[3 / 0.4,3.5 / 0.3]=[7.5,11.7] \approx 10,
$$

truly rogish compared to observations of a typical $A I \in[2,3]$ in the oceans. Coastal rogue waves can have a larger abnormality index, up to $A I \approx 5.5$, as discussed later. Finally, in November 2011, we created a portable bore-soliton-splash with also a circa tenfold amplification in a miniature wave channel of approximately $0.7 \mathrm{~m}$ long, $0.1 \mathrm{~m}$ 


\begin{tabular}{|c|c|c|c|c|c|c|}
\hline Case & $\begin{array}{l}h_{0}(\mathrm{~m}) \\
\pm 0.01 \mathrm{~m}\end{array}$ & $\begin{array}{l}h_{1}(\mathrm{~m}) \\
\pm 0.01 \mathrm{~m}\end{array}$ & $\begin{array}{c}H_{s} \\
\pm 0.05 \mathrm{~m}\end{array}$ & $\begin{array}{c}H_{r w} \\
\pm 0.5 \mathrm{~m} \\
\end{array}$ & $\begin{array}{c}\text { Peak } \\
\#\end{array}$ & Comments \\
\hline 1 & 0.32 & 0.67 & - & 0.6 & - & bore \\
\hline 2 & 0.38 & 0.74 & - & 2.5 & - & good splash \\
\hline 3 & $\underline{0.41}$ & $\underline{0.9}$ & 0.35 & 3.25 & $2^{\text {nd }}$ & $\begin{array}{l}\text { thin jet } \\
\text { cf. } 6 \& 8\end{array}$ \\
\hline 4 & 0.47 & 1.0 & 0.35 & 1 & $2^{\text {nd }}$ & bore \& low \\
\hline 5 & 0.41 & 1.02 & 0.40 & 1.5 & $1^{\text {st }}$ & bore \& low \\
\hline 6 & $\underline{0.41}$ & $\underline{0.9}$ & 0.35 & 3.5 & $2^{\text {nd }}$ & BSS cf. $3 \& 8$ \\
\hline 7 & 0.45 & 0.8 & 0.35 & 2.5 & $2^{\text {nd }}$ & good splash \\
\hline 8 & $\underline{0.41}$ & $\underline{0.9}$ & 0.35 & 3.5 & $2^{\text {nd }}$ & $\mathrm{BSS} \&++$ \\
\hline 9 & 0.43 & 0.9 & 0.45 & 1.8 & $1^{\text {st }}$ & collapsing into sheets \\
\hline
\end{tabular}

Table 2. Table with all experimental trials to establish the highest bore-soliton-splash (BSS). Cases 1-7 were trials on 27-09-2010 and Cases 8 and 9 took place on 30-09-2010. Reproducibility was verified with two nearly identical (underlined) Cases 3 and 6 on 27-09-2010 matching the (underlined) Case 8 on 30-09-2010. The excavator operator was instructed/trained to remove the sluice gate consistently by first pulling chains seen in Fig. 3b) taught before gate removal at $2.5 \mathrm{~m} / \mathrm{s}$. Cases 3,6 and 8 were nearly identical, as evidenced by video footage [48], with (slight) differences attributed to differences in rest levels $h_{0,1}$ and sluice-gate removal operation.

in width and 0.065 high, where we used $h_{0}=0.02 \pm 0.001 \mathrm{~m}$ and $h_{1} \approx 2 h_{0}$ to find $H_{r w}=0.2 \mathrm{~m}$. It again involved a solitary wave compound of a highest solitary wave followed by a second and third one of lower amplitudes. Perhaps surprisingly, nonlinear and inertial effects still dominate over friction and also over surface tension. A minisplash video is found online [48].

\section{Mathematical modelling}

The bore-soliton-splash involves a series of mathematical and fluid-mechanical ingredients: dispersion, nonlinearity, a turbulent spilling breaker and collapsing splash. Assuming incompressible fluid flow with a free surface, dispersion in a solitary wave is balanced by nonlinearity due to advection, while the hydraulic bore or spilling breaker highlights that this balance is temporarily and locally broken till turbulent dissipation reduces wave amplitude sufficiently to restore that balance, as we saw in Fig. 3c) to d). When the flow is in balance, the soliton compound and splash can be modelled with a single-valued free surface in a singly-connected domain till the apex of the splash is reached. Both spilling breaker and collapse of the splash are seen to involve multiplyconnected domains with bubbles and droplets.

We will start our modelling of the bore-soliton-splash cases for a smooth singlevalued free-surface and by using potential-flow equations and approximations thereof. Approximations used include a Benney-Luke pair/system of equations and the single, 
unidirectional KP equation in two horizontal spatial dimensions. These approximations have the advantage that dispersion is anomalously high which prohibits wave breaking and is therefore robust with the disadvantage being that outcomes during wave breaking will be less realistic. Numerical solutions are required to solve potential-flow and Benney-Luke equations in the actual wave channel, while exact solutions are available for the KP equation in an idealised domain for idealised settings. We will use variational principles and asymptotic theory to enhance numerical stability and robustness: our (novel) numerical techniques are direct, compatible space-time discretisations of relevant variational principles.

For potential-flow water waves, consider a free surface at $z=h(x, y, t)=H_{0}+$ $\eta(x, y, t)$ over a flat bottom at $z=0$ with vertical coordinate $z$, horizontal coordinates $x$ and $y$ as well as time $t$. Acceleration of gravity $g$ acts in the negative $z$-direction. Water at rest sits at $z=H_{0}$ and $\eta=\eta(x, y, t)$ is the deviation from this rest level $H_{0}$. Three-dimensional velocity $\boldsymbol{u}$ is approximated using a velocity potential $\phi=\phi(x, y, z, t)$ as $\boldsymbol{u}=\boldsymbol{\nabla} \phi$ with the gradient $\boldsymbol{\nabla}=\left(\partial_{x}, \partial_{y}, \partial_{z}\right)^{T}$. The horizontal part of the domain $\Omega_{h}$ is defined by a main channel of width $L_{x}$, with $x \in\left[0, L_{x}\right]$ and length $l_{y}(x)$, with $y \in\left[R(t), l_{y}(x)\right]$. The contraction is defined by $y=l_{y}(x)$ for $y \in\left[L_{y}-L_{c}, L_{y}\right]$, where

$$
l_{y}(x)=L_{y}-L_{c}\left|1-2 x / L_{x}\right|
$$

and the length of the contraction measured in the $y$-direction is $L_{c}$. The piston wavemaker $R(t)$ will be used later and for the solid wall at $x=0$ considered hitherto we take $R(t)=0$. Our derivation starts from Luke's [36] variational principle

$$
\begin{aligned}
0 & =\delta \int_{0}^{T} \mathcal{L}_{w}[\phi, \eta] \mathrm{d} t \\
& =\delta \int_{0}^{T} \iint_{\Omega_{h}} \int_{R(t)}^{H_{0}+\eta} \partial_{t} \phi+\frac{1}{2}|\nabla \phi|^{2}+\frac{1}{2}\left(\partial_{z} \phi\right)^{2}+g\left(z-H_{0}-\eta_{R}\right) \mathrm{d} z \mathrm{~d} x \mathrm{~d} y \mathrm{~d} t,
\end{aligned}
$$

modified to include a potential $\eta_{R}=\eta_{R}(x, t)$ modelling the removal of the sluice gate (cf. [5]) and with the horizontal domain extent $\Omega_{h}$ of the wave channel. Hereafter $\boldsymbol{\nabla}$ is the horizontal gradient with the $z$-derivatives explicitly indicated. Collecting all variables into the vector of unknowns

$$
\boldsymbol{U}=(\phi, \eta)^{T},
$$

variations of (3a) are defined as follows

$$
\delta \int_{0}^{T} \mathcal{L}_{w}[\boldsymbol{U}] \mathrm{d} t \equiv \lim _{\epsilon \rightarrow 0} \int_{0}^{T} \frac{\mathcal{L}_{w}[\boldsymbol{U}+\epsilon \delta \boldsymbol{U}]-\mathcal{L}_{w}[\boldsymbol{U}]}{\epsilon} \mathrm{d} t .
$$


The potential-flow water-wave equations resulting from (3a) read

$$
\begin{aligned}
& \nabla^{2} \phi+\partial_{z z} \phi=0 \quad \text { in } \quad \Omega_{h} \\
& \partial_{z} \phi=0 \quad \text { at } z=0 \text {, } \\
& \partial_{t} \eta+\nabla \phi \cdot \nabla \eta-\partial_{z} \phi=0 \quad \text { at } \quad z=H_{0}+\eta \text {, } \\
& \partial_{t} \phi+\frac{1}{2}|\nabla \phi|^{2}+\frac{1}{2}\left(\partial_{z} \phi\right)^{2}+g\left(\eta-\eta_{R}\right)=0 \quad \text { at } \quad z=H_{0}+\eta \text {, } \\
& \hat{\boldsymbol{n}} \cdot \nabla \phi=0 \quad \text { on } \quad \partial \Omega_{h},
\end{aligned}
$$

with $\hat{\boldsymbol{n}}$ the unit normal vector and $\partial \Omega_{h}$ the boundaries of the horizontal domain, i.e. any vertical walls - cf. the derivation in [36] with a minor change involving the sluice gate. To obtain the Benney-Luke system one can directly transform and simplify (3b), considered next.

\subsection{Benney-Luke water-wave modelling}

Pego and Quintero [43] and Bokhove and Kalogirou [5] introduced two transformations, here summarised in one transformation as

$$
\begin{gathered}
x=\frac{H_{0}}{\sqrt{\mu}} \hat{x}, \quad y=\frac{H_{0}}{\sqrt{\mu}} \hat{y}, \quad z=H_{0} \hat{z}, \quad t=\frac{H_{0}}{\sqrt{g H_{0} \mu}} \hat{t}, \quad \phi=\epsilon H_{0} \sqrt{\frac{g H_{0}}{\mu}} \hat{\phi} \\
\eta=\epsilon H_{0} \hat{\eta}, \quad \eta_{R}=\epsilon H_{0} \hat{\eta}_{R},
\end{gathered}
$$

with amplitude parameter $\epsilon=\alpha_{0} / H_{0} \ll 1$ and small dispersion parameter $\mu=$ $\left(H_{0} / l_{0}\right)^{2} \ll 1$ concerning long waves with a wavelength $l_{0}$ and an amplitude $\alpha_{0}$. After dropping the hats, the scaled velocity potential is expanded in terms of $\mu$, after some analysis resulting in the partial solution

$$
\phi(x, y, z, t)=\Phi(x, y, t)-\frac{\mu}{2} z^{2} \Delta \Phi(x, y, t)+\frac{\mu^{2}}{24} z^{4} \Delta^{2} \Phi(x, y, t)+\ldots
$$

with $\Delta=\nabla^{2}$. Substitution thereof into the variational principle (3b), after using the scaling (6), integration over $z$ and truncation at $O\left(\epsilon^{2} \mu, \epsilon^{3}\right)$, yields the variational principle for a (modified) Benney-Luke system on $\Omega_{h}$, i.e.

$$
\begin{aligned}
0=\delta \int_{0}^{T} & \iint_{\Omega_{h}} \eta \partial_{t} \Phi+\frac{\mu}{2} \nabla \eta \cdot \partial_{t} \nabla \Phi+\frac{1}{2}(1+\epsilon \eta)|\nabla \Phi|^{2}+\frac{1}{2} \eta^{2}-\eta_{R} \eta \\
& +\mu\left(\nabla q \cdot \nabla \Phi-\frac{3}{4} q^{2}\right) \mathrm{d} x \mathrm{~d} y \mathrm{~d} t \\
=\int_{0}^{T} & \iint_{\Omega_{h}}\left(\partial_{t} \Phi-\frac{\mu}{2} \partial_{t} \Delta \Phi+\frac{\epsilon}{2}|\nabla \Phi|^{2}+\eta-\eta_{R}\right) \delta \eta \\
& -\left(\partial_{t} \eta-\frac{\mu}{2} \partial_{t} \Delta \eta+\nabla \cdot((1+\epsilon \eta) \nabla \Phi)+\mu \Delta q\right) \delta \Phi \\
& -\mu\left(\Delta \Phi+\frac{3}{2} q\right) \delta q \mathrm{~d} x \mathrm{~d} y \mathrm{~d} t
\end{aligned}
$$


with detailed derivations found in $[43,5,21]$. Notice that we introduced an auxiliary variable $q$ to lower the order of the spatial derivatives, which introduction facilitates our finite-element discretisation. The equations of motion result from (8b) by assuming that the variations $\delta \eta, \delta \Phi, \delta q$ are arbitrary, and are given by setting the three equations in brackets equal to zero - see also [5, 21].

We will use a direct space-time discretisation of (8) by expanding the three variables using compact $C^{0}$-basis functions $\varphi_{l}(x, y)$, i.e.,

$$
\eta_{h}(x, y, t)=\eta_{l}(t) \varphi_{l}(x, y), \quad \phi_{h}(x, y, t)=\phi_{l}(t) \varphi_{l}(x, y), \quad q_{h}(x, y, t)=q_{l}(t) \varphi_{l}(x, y),
$$

with the subscript $(\cdot)_{h}$ indicating the spatial approximations made and (Einstein) summation over a finite discrete index $l=1, \ldots, N_{n}$, with $N_{n}$ the number of basis functions employed. After substitution of (9) into (8a) and integration in space, this reduces the variational principle to one in time only [21]. The temporal coefficients or variables are discretised by using a mixed (dis)continuous Galerkin finite-element expansion in time $[5,19]$, reducing the variational principle to an algebraic one for the remaining coefficients. Variations of this algebraic variational principle yield the (partially implicit) space-time discrete equations of motion, with our procedure guaranteeing that discrete conservation laws are conserved and that the energy is either conserved in time or displays only bounded oscillations proportional to a power of the time step $\Delta t$, and without any energy drift in time. We chose a time discretisation yielding a second-order Störmer-Verlet symplectic time integrator but our methodology facilitates other choices resulting in established and new time integrators. Hence, our simulations are expected to be accurate and robust with no artificial loss of wave amplitude. The final implementation is done in Firedrake, an automated system to deal with finite-element discretisations of partial differential equations, and our model is (freely) available online under an open license agreement [44]. Further details are found in $[5,21]$.

\section{Simulations:}

The Benney-Luke equations have been used to simulate Cases 8 and 9. The simulation of Case 9 is seen to be surprisingly good from the visual comparison between the photographic images and snapshots of the simulation in Fig. 5. The simulation of Case 9 shown are an improvement of the simulation in [5] because we have used better meshing with a symmetric mesh before the contraction, as seen in Fig. 11. The simulation of Case 8 does not compare well with the photographic images and video material. The primary reason is that simulations of Cases 8 and 9 with the Benney-Luke equations are fairly similar, as the comparison between these cases along the centreline of the wave tank in Fig. 6 reveal. Consequently, the reduction of the wave amplitude and, hence, wave speed of the first soliton due to wave breaking in Case 8 does not occur. The observed resonance between the first and second solitary wave in which the second wave exactly falls within the trough drawn by the reflection of the first wave in the contraction is absent in the simulation of Case 8. The resulting wave amplification seen in Case 8 is therefore also absent. The wave dispersion in Benney-Luke equations is too strong. 

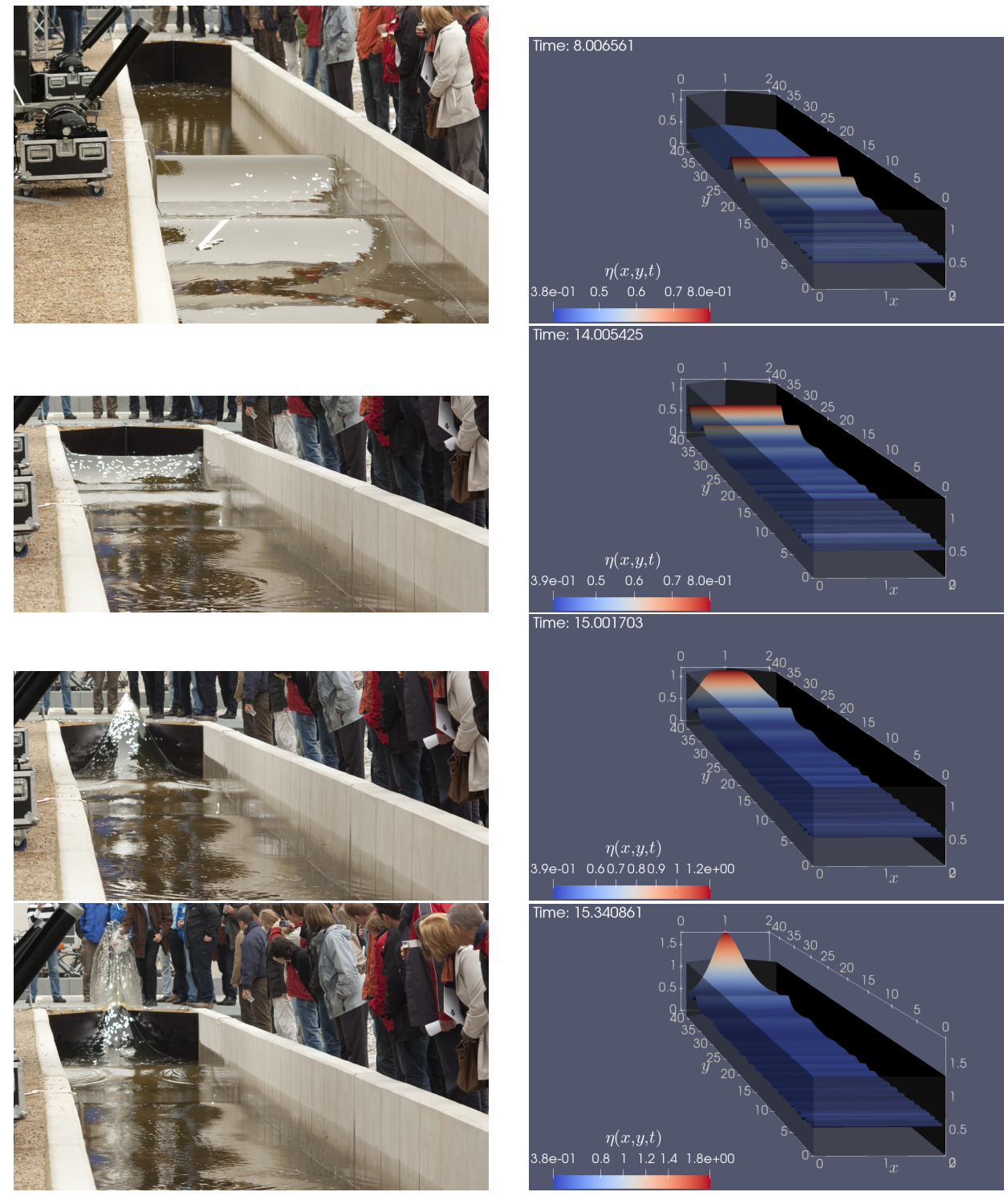

Figure 5. Snapshots of a soliton splash event Case 9. Left column: observations. Right column: numerical solution with $\mu=0.04, \epsilon=0.55$. When taking $h_{0}=0.43 \mathrm{~m}$ instead of $h_{0}=0.41 \mathrm{~m}$ as in Case 8 , with $h_{1}=0.9 \mathrm{~m}$ the same in both cases, no wave breaking occurs [4]. Photo times $t=8,14,15,15 \pm 0.5 \mathrm{~s}$ (relative) and simulation times $t=8,14,15,15.34 \mathrm{~s}$. Values displayed are in meters. The simulation involves $N_{k}=8010$ elements of which $N_{x} N_{y}=20 \times 390=7800$ elements lie in the regular part of the channel and $N_{x}\left(N_{x}+1\right) / 2=210$ elements in the triangular contraction. There are $N_{n}=8431$ nodes with $\left(N_{x}+1\right)\left(N_{y}+1\right)=8211$ nodes in the regular part of the channel and $N_{x}\left(N_{x}+2\right) / 2=220$ nodes in the triangular contraction. 

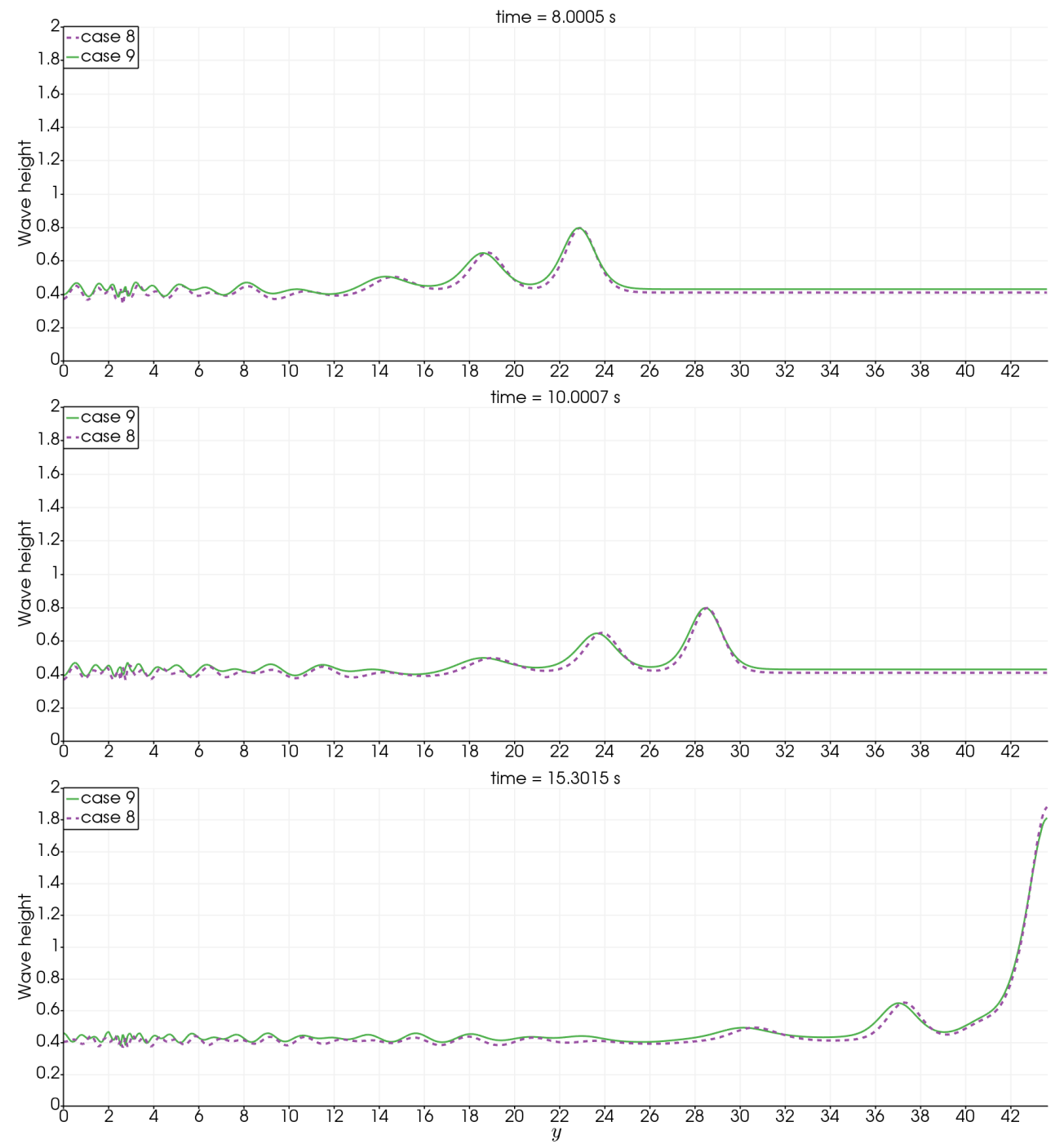

Figure 6. Snapshots at times $t=8.0,10.0,15.3 \mathrm{~s}$ of simulations for Cases 8 (dashed lines) and 9 (solid lines), presented as profiles along the centreline of the wavetank, i.e. at $x=L_{x} / 2=1 \mathrm{~m}$.

Either a potential-flow type model with parametrised wave breaking is required or a model with the dynamics of a water-air mixture, including the localised wave breaking inherent in such a two-phase model.

\section{Novel rogue-wave-energy device}

We have created, designed and tested a novel wave-energy device inspired by the boresoliton-splash event. Our rogue-wave-energy device has elements of three older and existing wave-energy devices [15]:

- The tapered channel or TapChan device; it consists of a tapered open channel which will enhance the wave amplitude such that at the channel end the waves overtop 


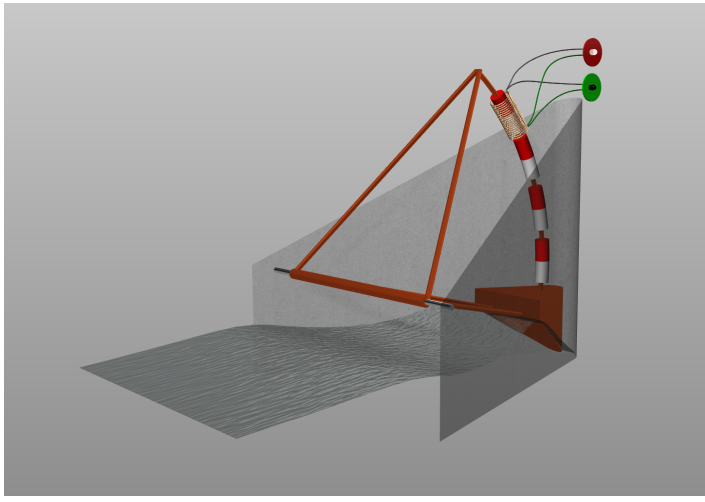

(a)

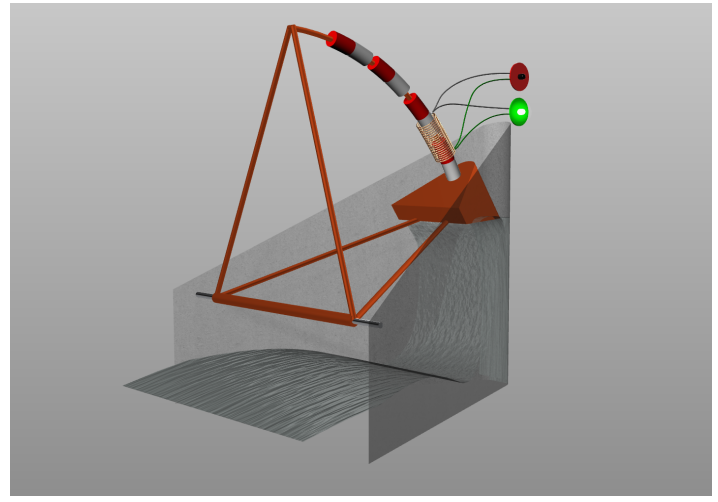

(b)

Figure 7. Sketches of our wave-energy device with its horizontal axel at the contraction entrance, its three-dimensional buoy in the contraction indicated in yellow/orange, attached to an induction motor, consisting of magnets on the arc moving through the hollow cylindrical coils indicated in green, as well as a green and red LED.

a levy and water flows into a reservoir. Elsewhere along the reservoir water flows down into a turbine to generate electricity via hydropower. A TapChan operated for a couple of years on a Norwegian island, bringing back electricity of $350 \mathrm{~kW}$ into the Norwegian grid, before it got damaged in a storm.

- The IPS wave buoy; it consists of a heaving buoy with a deep-lying piston moving into an anchored vertical shaft with a PTO (power take-off) mechanism to generate energy.

- The oscillating water column (OWC); it consists of a tapered channel in which the waves enter one open end of the channel, funnel and amplify in an enclosed converging section that turns into the vertical with a blow-hole at the top or the top side. Meanwhile, air compresses and decompresses by the rising and sinking wave leading to rapid air flow through the blow hole in which a Wells turbine is situated and generates electrical power when air flows in either direction.

Our device consists of a contracting channel with a wave-buoy constrained to move in only one dimension, either in the vertical by sliding along a guiding mast or along a slightly curved arc pivoting around a horizontal axel at the contraction entrance. Attached to the buoy is either another vertical mast or a curved mast, to which magnets are attached that can move through a series of coils when the buoy is heaving due to the wave motion. An artistic rendering of the second version of the wave-energy device is given in Fig. 7. The latter magnet-and-coil system comprises a magnetic-induction motor, cf. the one in the Faraday shaking light shown in Fig. 8b). Relative to the version with the two vertical masts, one moving and one fixed, it has the advantage that the buoy can be taken out of action in storms and that a rotating axel is mechanically more robust than mast-guide ball-bearings. Our device is intended to be part of a wavebreaker or dock since waves will be absorbed. 


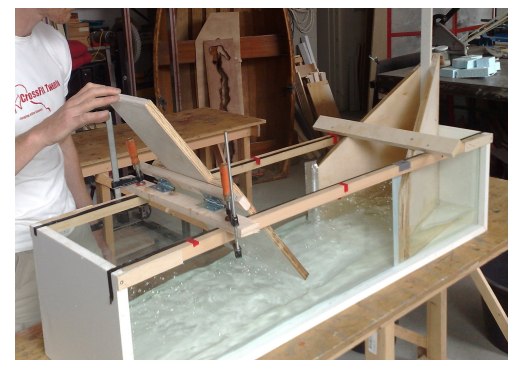

(a)

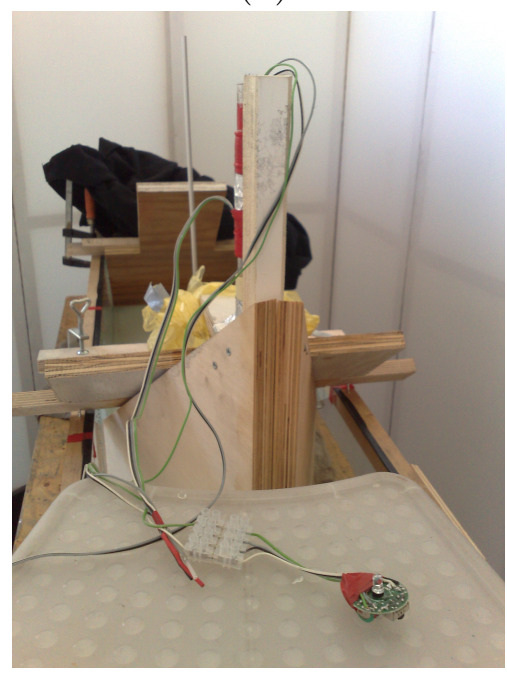

(c)

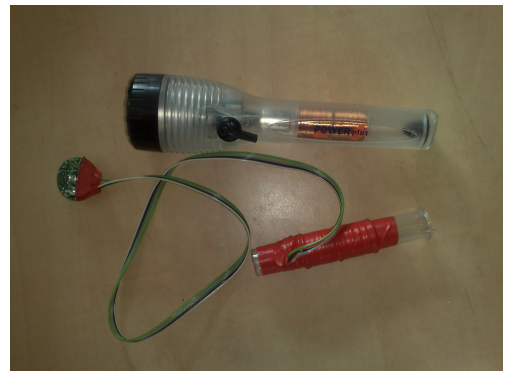

$(b)$

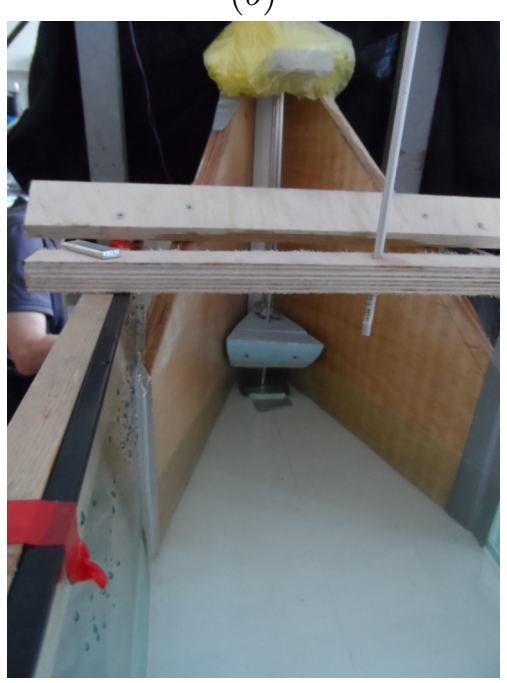

(d)

Figure 8. Overview of the working proof-of-principle of our new wave-energy device, here powering one LED: a) wavetank with wavemaker, powered by OB, and contraction; b) two Faraday shaking lights, one entire and one deconstructed with the magnets put into and the coils wrapped around a plastic tube; c) the tube guiding the magnet with its surrounding coils and wires leading to the LED; and, d) the unit of contraction, guiding mast and buoy-mast unit, at rest.

In 2013 the "Berkeley wedge" wave-energy device was patented [37]. It is a waveabsorbing wedge moving on rails against a vertical wall attached to an induction motor. It is similar to our wave-energy device but has no wave-amplitude enhancing contraction like in the TapChan and OWC devices. During storms the Berkeley wedge is sunk into the water to protect it from damage. The Berkeley wedge operates in essence like a wavemaker reversed in time, following the principle that a good wave-energy device can also be good wavemaker, when time is reversed or energy is put in rather than being generated.

Before we advanced to any mathematical modelling, we built and tested a proof-ofprinciple of our device to assess its viability in the summer of 2013. Our experimental set-up consisted of a straightforward wave-tank with a hand-driven wavemaker, Fig. 8a), a shaped foam buoy with a mast topped by two magnets moving through a tube with four fixed coils, Fig. 8c), the latter parts coming out of two deconstructed shake or 


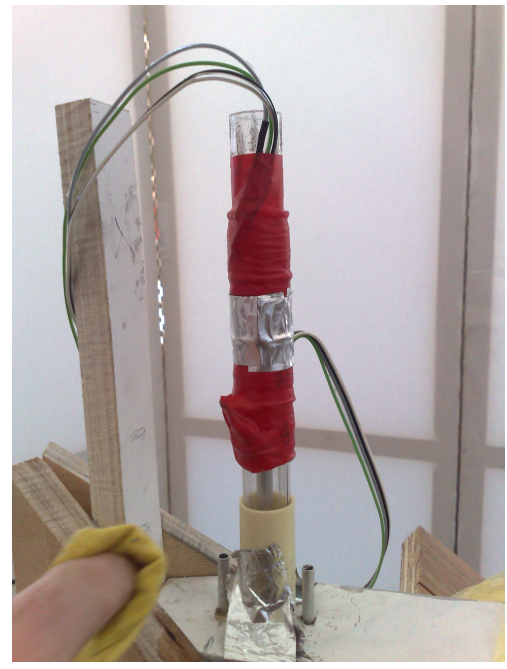

(a)

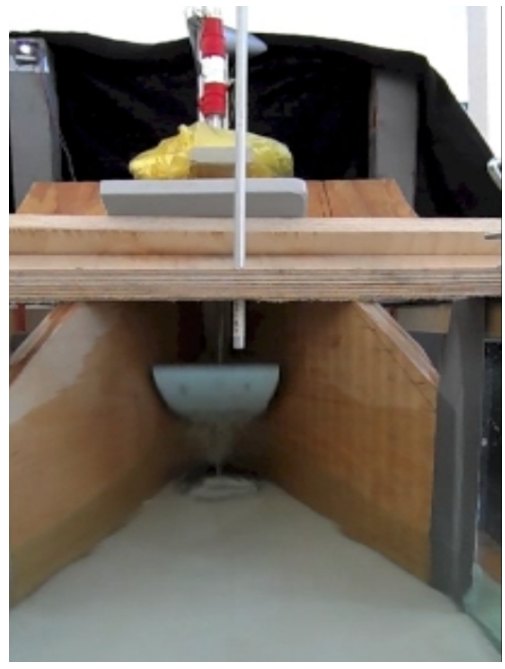

(b)

Figure 9. Details of our new wave-energy device: a) The magnetic-induction motor consisting of the hollow tube with its four sets of coils and the LED light through which the magnets on top of the buoy-mast move; and, b) the blinking LED light (seen as the white flash at the top left) while the buoy is elevated by a wave to its top position.

Faraday flashlights $[20,25,38]$, Fig. 8b), with the buoy constrained to glide along a fixed, guiding mast. The shape of the buoy is close to a simplex with a slightly rounded and slanted bottom face and a flat top face, Fig. 8d). To the induction motor, either one LED was connected to demonstrate the power output or an Arduinoscope (a handmade oscilloscope using Arduino technology) to measure power output. Photographs of the set-up are given in Figs. 8 and 9. We powered one LED but, given the AC-power generated, two (sets of) LEDs will be used in the mathematical model derived in the next section (see also [28, 29]). Two (sets of) LEDs, circuited in parallel yet operating for currents in opposite directions, harness twice the wave energy into light $\|$.

\subsection{Monolithic modelling of waves, buoy motion and linear induction actuator}

A comprehensive mathematical model of the wave-energy device will be developed next within a domain constructed to reproduce an existing small-scale wavetank at the University of Leeds, which is a larger tank than the one used for the proof-of-principle. The numerical wavetank has a piston wavemaker on its left side, consists of a channel with a flat bottom at $z=0$ that ends in a $\mathrm{V}$-shaped contraction at the right end of the channel, cf. Fig. 10, as described in $\S 3$ and equation (2). A wave-energy buoy, here constrained to move only in the vertical, resides in the corner of the contraction. The shape of the buoy is described next.

The buoy is a simplex with a flat top triangular face and has a slanted front face converging into one point at the bottom. The two remaining faces of the simplex align

|| Movie of 2013 proof-of-principle design/test: https://www. youtube.com/watch?v=SZhe_SOxBWo 
(a)

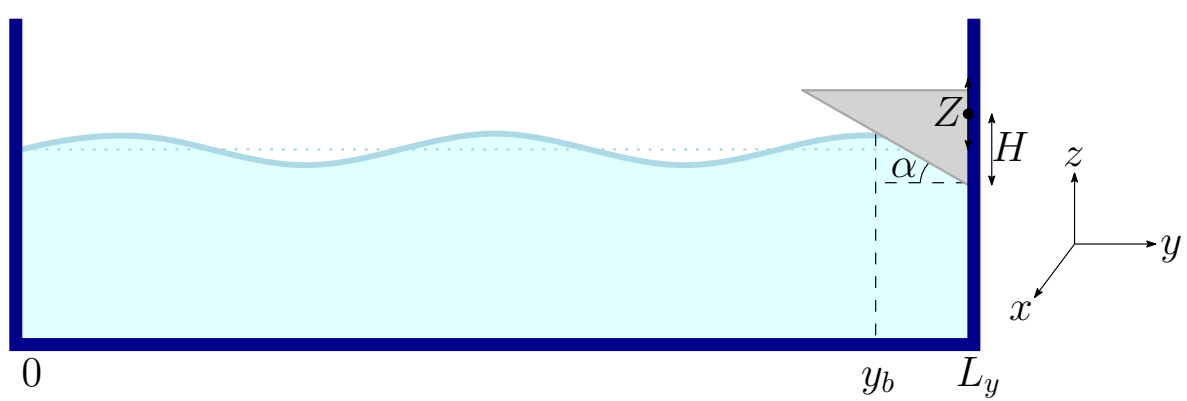

(b)

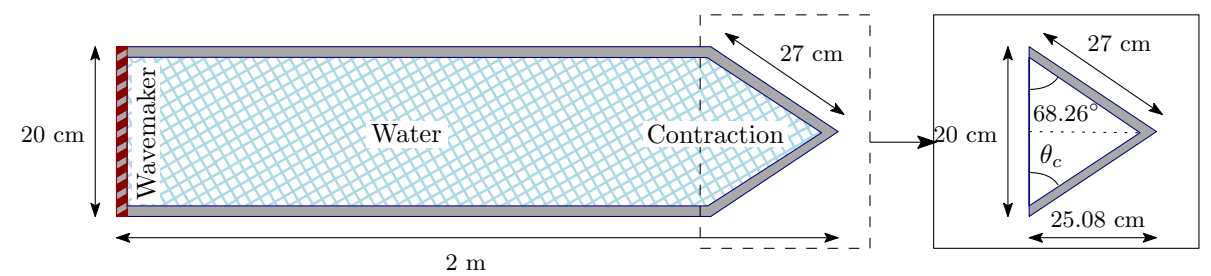

Figure 10. a) Sketch of a cross-section at the centreline of the wavetank with a contraction at its right end, and b) a top-view with $L_{y}=2 \mathrm{~m}, L_{x}=0.2 \mathrm{~m}$ and $L_{c}=0.2508 \mathrm{~m}$.

with the vertical walls of the $\mathrm{V}$-shaped contraction. The slanted face is tilted to the vertical, as shown in the cross-section at $x=L_{x} / 2$ in Fig. 10a). The buoy motion can be described by the position of its centre of mass $Z=Z(t)$ and corresponding velocity $W=W(t)=\mathrm{d} Z / \mathrm{d} t$. Given this simplex geometry and assuming its flat top face stays dry, the wetted buoy's height above the flat bottom is located at

$$
z=h_{b}(x, y ; Z(t))=Z(t)-H_{k}-\tan \alpha\left(y-L_{y}\right)
$$

Here, $\alpha$ is the angle between the hull bottom and the horizontal, $L_{y}$ is the maximum length along $x=L_{x} / 2$ of the domain with the wavemaker at its rest position $y=0$ and $H_{k}>0$ is the vertical distance between the centre of mass $Z(t)$ and the keel of the buoy (see Fig. 10a). The keel of this V-shaped buoy therefore lies at $(x, y, z)=$ $\left(L_{x} / 2, L_{y}, Z-H_{k}\right)$. The waterline point $y_{b}\left(x=L_{x} / 2, t\right)$ at the centreline $x=L_{x} / 2$ shown in Fig. 10 is defined as the point where the water meets the buoy. The overall waterline of the buoy is denoted by $y=y_{b}(x, t)$ and parameterised by $x$. At this waterline $y_{b}(x, t)$, for every $x$ within the contraction, which $x$-interval varies over time, the height $h=h(x, y, t)$ of the free surface of the fluid equals the buoy's surface height. That is $h\left(x, y_{b}^{-}(x, t), t\right)=h_{b}\left(x, y_{b}^{+}(x, t) ; Z(t)\right)$, with the appropriate limits indicated. Taking the variation of this expression one finds that

$$
\begin{gathered}
\delta h\left(x, y_{b}^{-}, t\right)=\delta h_{b}\left(x, y_{b}^{+}, t\right) \Leftrightarrow \\
\left.\delta h\right|_{y_{b}^{-}}+\left.\frac{\partial h}{\partial y}\right|_{y_{b}^{-}} \delta y_{b}=\left.\delta h_{b}\right|_{y_{b}^{+}}+\left.\frac{\partial h_{b}}{\partial y}\right|_{y_{b}^{+}} \delta y_{b} \quad \Leftrightarrow \quad \delta y_{b}=\frac{\delta Z-\left.\delta h\right|_{y_{b}^{-}}}{\left.\frac{\partial h}{\partial y}\right|_{y_{b}^{-}}+\tan \alpha}
\end{gathered}
$$


Table 3. Physical parameters used in three-dimensional numerical calculations for the wave-buoy system, including wavetank dimensions, buoy's mass and physical properties of water at a room temperature of $25^{\circ} \mathrm{C}$.

\begin{tabular}{ll}
\hline Channel width & $L_{x}=0.2 \mathrm{~m}$ \\
Channel length & $L_{y}=2.0 \mathrm{~m}$ \\
Channel height & $L_{z}=0.2 \mathrm{~m}$ \\
Rest water depth & $H_{0}=0.1 \mathrm{~m}$ \\
Buoy mass & $M=0.05 \mathrm{~kg}$ \\
Density of water & $\rho=997 \mathrm{~kg} / \mathrm{m}^{3}$ \\
Gravity & $g=9.81 \mathrm{~m} / \mathrm{s}^{2}$ \\
\hline
\end{tabular}

which implies that $y_{b}$ is not an independent variable in the problem.

The rest position of the buoy is directly determined geometrically via Archimedes' principle given the mass $M$ and rest centre of mass $\bar{Z}$ of the buoy, as follows. The angle $\theta_{c}$, defined by $\tan \theta_{c}=2 L_{c} / L_{x}$, with $\theta_{c}=68.26^{\circ}$ presently, is the angle between the opening of the contraction and the line across the contraction (as shown in the magnified right-hand-side panel in Fig. 10). A summary of the dimensions of the existing wavetank as well as other relevant physical parameters used in the numerical calculations can be found in Table 3. At rest, $y_{b}=L_{b}$ and given that $\tan \theta_{c}=2 L_{c} / L_{x}=\left(L_{y}-L_{b}\right) / X_{b}$, the length of the waterline is $2 X_{b}=2\left(L_{y}-L_{b}\right) / \tan \theta_{c}$, such that the submerged part of the buoy is a smaller simplex, isomorphic to the entire buoy simplex, defined by the following four points:

$$
\begin{aligned}
& \mathbf{v}_{0}=\left(\frac{1}{2} L_{x}, L_{y}, H_{0}\right)^{T}, \quad \mathbf{v}_{1}=\left(\frac{1}{2} L_{x}-X_{b}, L_{b}, H_{0}\right)^{T}, \\
& \mathbf{v}_{2}=\left(\frac{1}{2} L_{x}+X_{b}, L_{b}, H_{0}\right)^{T}, \quad \mathbf{v}_{3}=\left(\frac{1}{2} L_{x}, L_{y}, \bar{Z}-H_{k}\right)^{T} .
\end{aligned}
$$

The volume $V_{b}$ of the submerged part of the buoy (cf. wikipedia) is then the displaced water mass divided by the density of water

$$
V_{b}=\frac{M}{\rho_{0}}=\frac{1}{6}\left|\operatorname{det}\left(\mathbf{v}_{1}-\mathbf{v}_{0}, \mathbf{v}_{2}-\mathbf{v}_{0}, \mathbf{v}_{3}-\mathbf{v}_{0}\right)\right|=\frac{1}{3} \frac{\left(H_{0}+H_{k}-\bar{Z}\right)^{3}}{\tan \theta_{c} \tan ^{2} \alpha}
$$

given that $\tan \alpha=\left(H_{0}+H_{k}-\bar{Z}\right) /\left(L_{y}-L_{b}\right)$ (calculated via $(10)$ for $\left.(x, z)=\left(L_{y}, H_{0}\right)\right)$. Consequently, for this three-dimensional tetrahedral buoy, the rest position of the buoy's centre of mass is thus found to be

$$
\bar{Z}=H_{0}+H_{k}-\sqrt[3]{\frac{3 M \tan \theta_{c} \tan ^{2} \alpha}{\rho_{0}}}, \quad \text { and } \quad L_{b}=L_{y}-\sqrt[3]{\frac{3 M \tan \theta_{c}}{\rho_{0} \tan \alpha}} .
$$

Attached to the buoy is a vertical mast with two magnets on top, moving through a set of coils, whose constrained and vertical movement by induction comprises the actuator. Magnet and mast are all included in the overall weight $M$ of the buoy. The 
current $I=I(t)$ is the derivative of the electrical charge $Q=Q(t)$, i.e. $I=\dot{Q}$. The coils have an overall inductance of $L_{i}$. Rather than using the current $I(t)$, we use the conjugate momentum $P_{Q}=P_{Q}(t)$ as primary variable, defined in Appendix A by $P_{Q}=L_{i} \dot{Q}-K(Z)$ with

$$
K(Z)=\int^{Z} \gamma G(\hat{Z}) \mathrm{d} \hat{Z},
$$

$\gamma=2 \pi a^{2} \mu N / L$, magnetic dipole momentum $\mu$ of the magnet, $a$ the radius of the coils, $N$ the number of coil windings per metre, $L$ the length of the coils as well as a function $G(Z)$ defined in (A.10f). This function $G(Z)$ depends on the length of the mast $H_{m}$, the length $L_{m}$ and radius of the cylindrical magnet and the placement of the coils at $z \in\left[\bar{Z}+\left(1+\alpha_{h}\right) H_{m}-L / 2, \bar{Z}+\left(1+\alpha_{h}\right) H_{m}+L / 2\right]$ with $0<\alpha_{h}<1$. The model of the magnentic-induction actuator is developed in Appendix A.

Hence, we can now formulate a comprehensive, or monolithic, variational principle of the entire, coupled water-wave problem, buoy motion and magnetic-induction actuator, as follows

$$
\begin{aligned}
& 0=\delta \int_{0}^{T} \mathcal{L}\left[D, \phi, h, \phi_{s}, Z, W, Q, P_{Q}, p, \lambda\right] \mathrm{d} t \\
& \equiv \delta \int_{0}^{T} \rho_{0} \int_{0}^{L_{x}} \int_{R(t)}^{l_{y}(x)} \int_{0}^{h(x, y, t)} D \partial_{t} \phi \mathrm{d} z \mathrm{~d} y \mathrm{~d} x-M W \dot{Z}-P_{Q} \dot{Q}+\mathcal{H} \mathrm{d} t \\
& \equiv \delta \int_{0}^{T} \rho_{0} \int_{0}^{L_{x}} \int_{R(t)}^{l_{y}(x)} \int_{0}^{h(x, y, t)} D \partial_{t} \phi+\frac{1}{2} D|\nabla \phi|^{2}+g D\left(z-H_{0}\right)+p(D-1) \mathrm{d} z \mathrm{~d} y \mathrm{~d} x \\
& \quad+\rho_{0}^{L_{x}} \int_{R(t)}^{l_{y}(x)} \lambda\left(h-h_{b}\right) \Theta\left(y-y_{b}(x, t)\right) \mathrm{d} x \mathrm{~d} y \\
& \quad \frac{-M W \dot{Z}-P_{Q} \dot{Q}+\frac{1}{2} M W^{2}+M g Z+\frac{1}{2} \frac{\left(P_{Q}+K(Z)\right)^{2}}{L_{i}} \mathrm{~d} t,}{}
\end{aligned}
$$

with $\phi_{s}=\phi(x, y, h(x, y, t), t)$ the velocity potential evaluated at the free surface. Herein, a scaled pressure $p=p(x, y, z, t)$ acts as Lagrange multiplier to impose the incompressibility constraint $D-1=0$ of a scaled density $D=D(x, y, z, t)$ such that the density $\rho(x, y, z, t)=\rho_{0} D(x, y, z, t)$, cf. [10]. Moreover, the single-valued free water surface at $z=h(x, y, t)$ with water depth $h=h(x, y, t)$ is constrained (underlined terms in (16)) to be the dynamic shape of the wavebuoy by using the Lagrange multiplier $\lambda(x, y, t)$ over the wetted part $y>y_{b}(x, t)$ of the wavebuoy hull - see also [28]. We have used the Heaviside function $\Theta\left(y-y_{b}\right)$, zero for $y<y_{b}$ and unity for $y \geq y_{b}$, to single out this wetted part of the hull. The key reason to include the scaled density $D$ and impose incompressibility condition $D-1=0$ weakly is that variational principle (16) mathematically yields the boundary condition on $\lambda$ at the waterline, as a consistency component of the entire formulation.

As before, collecting all variables into the vector of unknowns

$$
\boldsymbol{U}=\left(D, \phi, h, \phi_{s}, Z, W, Q, P_{Q}, p, \lambda\right)^{T},
$$


variations of (16) are defined as in (4). Most variations of (16) emerge in a straightforward manner; only the variations involving the terms $D \partial_{t} \delta \phi, D \boldsymbol{\nabla} \phi \cdot \boldsymbol{\nabla}(\delta \phi)$ and a comprehensive term involving the variations of the free surface $\delta h$ in the upper integration limit are more complicated and require integration by parts in time and the use of Gauss' law - see [10, 18] for more details. Given these hints while leaving further derivation details to the reader, variation of (16) yields the following, fully nonlinear, equations of motion,

$$
\begin{aligned}
& \delta D: \quad \partial_{t} \phi+\frac{1}{2}|\nabla \phi|^{2}+g\left(z-H_{0}\right)+p=0 \\
& \delta \phi: \quad \partial_{t} D+\nabla \cdot(D \nabla \phi)=0 \\
& \delta p: \quad D=1 \\
& \delta \lambda: \quad h-h_{b}=0 \text { for } y \geq y_{b}(x, t) \\
& \delta \phi_{s}: \quad \partial_{t} h+\nabla_{H} \phi \cdot \nabla h=\partial_{z} \phi \quad \text { at } \quad z=h(x, y, t) \\
& \delta h: \quad \partial_{t} \phi+\frac{1}{2}|\nabla \phi|^{2}+g\left(h-H_{0}\right)+\lambda \Theta\left(y-y_{b}(x, y, t)\right)=0 \quad \text { at } \quad z=h(x, y, t) \\
& \delta \phi_{R}: \quad \dot{R}=\partial_{x} \phi \quad \text { at } \quad x=R(t) \\
& \delta W: \quad \dot{Z}=W \\
& \delta Z: \quad M \dot{W}+M g+\frac{\gamma G(Z)}{L_{i}}\left(P_{Q}+K(Z)\right) \\
& -\rho_{0} \int_{0}^{L_{x}} \int_{R(t)}^{l_{y}(x)} \lambda \Theta\left(y-y_{b}(x, t)\right) \mathrm{d} x \mathrm{~d} y=0 \\
& \delta P_{Q}: \quad \dot{Q}=\frac{\left(P_{Q}+K(Z)\right)}{L_{i}} \equiv I \\
& \delta Q: \quad \dot{P}_{Q}=0
\end{aligned}
$$

with $\phi_{R}=\phi(R(t), y, z, t)$ the velocity potential evaluated at the wavemaker and $\nabla_{H}=\left(\partial_{x}, \partial_{y}\right)^{T}$ the horizontal gradient operator. Evaluation of (17a) at the free surface $z=h$ and subtraction of $(17 \mathrm{f})$ yields that

$$
p=0 \quad \text { for } y<y_{b}(x, t), \quad \text { and } \quad p=\lambda \text { for } y \geq y_{b}(x, t)
$$

such that $y=y_{b}(x, t)$ at the waterline on the wavebuoy, from which it necessarily follows that $\lambda\left(x, y_{b}(x, t), t\right)=0$.

A straightforward way to model the energy harvest is the use of two (sets of) LED diodes in parallel but positioned in opposite directions such that only one (set of) LED(s) is active at one time. Using the Shockley equation, as model for the LED-voltage-current relationship for current flow in either direction, yields that the voltage

$$
V_{s}(I)=-\operatorname{sign}(I) n_{q} V_{T} \ln \left(|I| / I_{s a t}+1\right)
$$

is really a function of $I=I(t)$; the sign-function and absolute value $|I|$ used ensure operation and damping for currents in either direction. Since such a LED-model leads 
to damping it is less common to include it a priori in the variational principle and damping/loading is added a posteriori to the model by substitution of (19) for $V_{s}(t)$. Parameters in the Shockley model for LEDs include the saturation current $I_{\text {sat }}$, the quality factor $n_{q}$ and the thermal voltage $V_{T}$. In addition, we added resistance terms $-\left(R_{c}+R_{i}\right) I$, with a resistance $R_{i}$ of the wiring to the LEDs as well as a resistance $R_{c}$ of the coils, in the equation for $P_{Q}$, to model losses for the circuit and LED diodes combined. Instead of $\dot{P}_{Q}=0$ in (17), one then obtains

$$
\dot{P}_{Q}=-\left(R_{c}+R_{i}\right) I-\operatorname{sign}(I) n_{q} V_{T} \ln \left(\frac{|I|}{I_{s a t}}+1\right) \quad \text { with } \quad I=\left(P_{Q}+K(Z)\right) / L_{i} .
$$

The total electrical power output $P$ is then the time integral $P=\int_{0}^{T} I(t) V_{s}(t) \mathrm{d} t$, with $V_{s}(t)$ the voltage across the LEDs.

In the rest state, we saw that the straight waterline lies at $y=L_{b}$ with rest depth $H(x, y)=H_{0}$ for $y<L_{b}$, while for $y \geq L_{b}$ a rest depth $H(x, y)$ as well as (rest-state) Lagrange multiplier $\Lambda(x, y)$ are defined by

$$
H(x, y)=\bar{Z}-H_{k}-\tan \alpha\left(y-L_{y}\right) \text { and } \Lambda(x, y)=g\left(H_{0}+H_{k}-\bar{Z}+\tan \alpha\left(y-L_{y}\right)\right),
$$

with the centre of mass $\bar{Z}$ of the buoy at rest. Hence,

$$
\frac{\partial \Lambda}{\partial y}=g \tan \alpha
$$

To linearise the equations of motion (17), we consider the following decomposition of variables into rest-state and (small-amplitude) perturbations

$$
\begin{aligned}
\phi(x, y, z, t) & =\tilde{\phi}(x, y, z, t), \quad D(x, y, z, t)=1+\tilde{D}(x, y, z, t), \\
h(x, y, t) & =H(x, y)+\eta(x, y, t), \quad p(x, y, z, t)=g\left(H_{0}-z\right)+\tilde{p}(x, y, z, t), \\
\lambda(x, y, t) & =\Lambda(x, y)+\tilde{\lambda}(x, y, t), \quad y_{b}(x, t)=L_{b}+\tilde{y}_{b}(x, t), \quad Z(t)=\bar{Z}+\tilde{Z}(t), \\
W(t) & =\tilde{W}(t), \quad Q(t)=\tilde{Q}(t), \quad P_{Q}(t)=-K(\bar{Z})+\tilde{P}_{Q}(t),
\end{aligned}
$$

the latter relation taken such that the rest current $\bar{I}=0$ since $P_{Q}=L_{i} I-K(Z)$. Upon linearising, the moving domain becomes a fixed domain $y \in\left[0, l_{y}(x)\right]$ with a fixed waterline at $y=L_{b}$. After a Taylor expansion, the waterline condition for $\tilde{\lambda}$ at $y=L_{b}$ becomes

$$
0=\left.\lambda\left(x, y_{b}, t\right) \approx \frac{\partial \Lambda(x, y)}{\partial y}\right|_{y=L_{b}} \tilde{y}_{b}+\tilde{\lambda}\left(x, L_{b}, t\right),
$$

where we used that $\Lambda\left(x, L_{b}\right)=0$ by definition and omitted quadratic and higher-order terms in the perturbation variables. Expansion of the waterline condition (11) leads to an explicit expression for $\tilde{y}_{b}(x, t)$ in terms of $\tilde{Z}(t)$ and the free surface at the linearised waterline $\eta\left(x, L_{b}^{-}, t\right)$; to wit

$$
\tilde{y}_{b}(x, t)=\frac{\left(\tilde{Z}(t)-\eta\left(x, L_{b}^{-}, t\right)\right)}{\tan \alpha} .
$$


By combining (24) and (25), we obtain the desired boundary condition at the linearised waterline, i.e.

$$
\tilde{\lambda}\left(x, L_{b}, t\right)=g\left(\eta\left(x, L_{b}^{-}, t\right)-\tilde{Z}(t)\right) .
$$

It means that $\tilde{\lambda}\left(x, L_{b}, t\right)$ is not an independent variable at $y=L_{b}$.

The final simplifications are that we consider the system in both the linear and shallow-water limits, yielding that the velocity potential $\phi=\phi(x, y, t)$ is a function of only the horizontal coordinates and time, now with $\boldsymbol{\nabla}=\left(\partial_{x}, \partial_{y}\right)^{T}$. The equations of motion and induction in these linear, shallow-water limits become

$$
\begin{aligned}
& \eta-\tilde{Z}=0 \quad \text { for } y \geq L_{b}, \quad \dot{R}=\partial_{x} \tilde{\phi} \quad \text { at } \quad x=0 \\
& \partial_{t} \eta+\nabla \cdot(H \nabla \tilde{\phi})=0 \\
& \partial_{t} \tilde{\phi}+g \eta+\tilde{\lambda} \Theta\left(y-L_{b}\right)=0 \\
& \dot{\tilde{Z}}=\tilde{W} \\
& M \dot{\tilde{W}}+\gamma G(\bar{Z}) \frac{\left(\tilde{P}_{Q}+\gamma G(\bar{Z}) \tilde{Z}\right)}{L_{i}}-\rho_{0} \int_{0}^{L_{x}} \int_{0}^{l_{y}(x)} \tilde{\lambda} \Theta\left(y-L_{b}\right) \mathrm{d} y \mathrm{~d} x=0 \\
& \dot{\tilde{Q}}=\frac{\left(\tilde{P}_{Q}+\gamma G(\bar{Z}) \tilde{Z}\right)}{L_{i}} \\
& \dot{\tilde{P}}_{Q}=-\left(R_{c}+R_{i}+\frac{n_{q} V_{T}}{I_{s a t}}\right) \frac{\left(\tilde{P}_{Q}+\gamma G(\bar{Z}) \tilde{Z}\right)}{L_{i}} \\
& \nabla \cdot(H \nabla \tilde{\lambda})-\frac{\rho_{0}}{M} \int_{0}^{L_{x}} \int_{0}^{l_{y}(x)} \tilde{\lambda} \Theta\left(y-L_{b}\right) \mathrm{d} y \mathrm{~d} x=-\nabla \cdot(g H \nabla \eta)-\frac{\gamma}{M} G(\bar{Z}) \tilde{I} \\
& \text { for } y \geq L_{b},
\end{aligned}
$$

where we have added the effective circuit and coil resistances $R_{i}$ and $R_{c}$ and linearised (around $I=0$ and by using $\bar{Z}$ ) model of the LED-light, as well as a consistency equation, with (26) and $\hat{\boldsymbol{n}} \cdot \boldsymbol{\nabla} \tilde{\phi}=0$ at the fixed, vertical walls. Note that from (27f), $\tilde{I}=\left(\tilde{P}_{Q}+\gamma G(\bar{Z}) \tilde{Z}\right) / L_{i}$. When we rework $(27 \mathrm{~g})$ and take $\dot{\tilde{I}}$ to be negligible, then the linearisation is seen to lead to a damping term proportional to $\dot{\tilde{Z}}=\tilde{W}$ in the momentum equation, cf. [11, 12]. In addition, the linearisation of the LED-model (20) is seen to lead to an effective resistance $n_{q} V_{T} / I_{\text {sat }}$, with linearised LED voltage $\tilde{V}_{s}(\tilde{I})=n_{q} V_{T} / I_{\text {sat }} \tilde{I}$. The (linearised) electrical power output $\tilde{P}(t)$ is calculated as a function of time and is given by $\tilde{P}=\left(\tilde{V}_{s}+\tilde{V}_{r}\right) \tilde{I}$, with $\tilde{V}_{r}=\left(R_{c}+R_{i}\right) \tilde{I}$ the lost voltage due to circuit resistance.

A consistency equation arises by taking the time derivative of the primary constraint $\eta-\tilde{Z}=0$, which leads to a secondary constraint

$$
\boldsymbol{\nabla} \cdot(H \boldsymbol{\nabla} \tilde{\phi})+\tilde{W}=0
$$

upon using two of the equations of motion to eliminate time derivatives. Subsequently taking the time derivative of this secondary constraint (28), while using two other equations of motion to eliminate the emerging time derivatives, yields the elliptic equation (27h) for $\tilde{\lambda}$. 


\subsection{Time discretisation of the linearised system}

The time discretisation of the eight main equations in (27), i.e. in a count excluding boundary conditions, needs to be such that a time discretisation of the first seven equations is equivalent to, and consistent with a time discretisation of the last seven equations, given that there are seven unknowns. The chosen time discretisation is a symplectic Euler one [33, 19], given the conjugate pairs of variables $\{\tilde{\phi}, \eta\},\{\tilde{W}, \tilde{Z}\},\left\{\tilde{P}_{Q}, \tilde{Q}\right\}$. Hence, the time-discrete system with discrete time levels $t^{n}$ and $t^{n+1}=t^{n}+\Delta t$ reads

$$
\begin{aligned}
& \eta^{n+1}-\tilde{Z}^{n+1}=0 \quad \text { for } \quad y \geq L_{b} \\
& \frac{\left(\tilde{\phi}^{n+1}-\tilde{\phi}^{n}\right)}{\Delta t}+g \eta^{n}+\tilde{\lambda}^{n} \Theta\left(y-L_{b}\right)=0 \\
& \frac{\left(\tilde{W}^{n+1}-\tilde{W}^{n}\right)}{\Delta t}+C_{1} G(\bar{Z})\left(\tilde{P}_{Q}^{n}+\gamma G(\bar{Z}) \tilde{Z}^{n}\right)-C \int_{0}^{L_{x}} \int_{0}^{l_{y}(x)} \tilde{\lambda}^{n} \Theta\left(y-L_{b}\right) \mathrm{d} y \mathrm{~d} x=0 \\
& \frac{\left(\tilde{P}_{Q}^{n+1}-\tilde{P}_{Q}^{n}\right)}{\Delta t}=-C_{2}\left(\tilde{P}_{Q}^{n+1}+\gamma G(\bar{Z}) \tilde{Z}^{n}\right) \\
& \frac{\left(\tilde{Z}^{n+1}-\tilde{Z}^{n}\right)}{\Delta t}=\tilde{W}^{n+1} \\
& \frac{\left(\tilde{Q}^{n+1}-\tilde{Q}^{n}\right)}{\Delta t}=\frac{\left(\tilde{P}_{Q}^{n+1}+\gamma G(\bar{Z}) \tilde{Z}^{n}\right)}{L_{i}}, \\
& \frac{\left(\eta^{n+1}-\eta^{n}\right)}{\Delta t}+\nabla \cdot\left(H \nabla \tilde{\phi}^{n+1}\right)=0 \\
& \text { with }\left.\quad \partial_{x} \tilde{\phi}^{n+1}\right|_{x=0, L}=0,\left.\quad \partial_{x} \tilde{\phi}^{n+1}\right|_{x=0}=\dot{R}^{n+1} \\
& \boldsymbol{\nabla} \cdot\left(H \boldsymbol{\nabla} \tilde{\lambda}^{n}\right)-C \int_{0}^{L_{x}} \int_{0}^{l_{y}(x)} \tilde{\lambda}^{n} \Theta\left(y-L_{b}\right) \mathrm{d} y \mathrm{~d} x=-g \boldsymbol{\nabla} \cdot\left(H \boldsymbol{\nabla} \eta^{n}\right) \\
& -C_{1} G(\bar{Z})\left(\tilde{P}_{Q}^{n}+\gamma G(\bar{Z}) \tilde{Z}^{n}\right) \quad y \geq L_{b} \\
& \text { with } \quad \tilde{\lambda}^{n}\left(x, L_{b}, t\right)=g\left(\eta^{n}\left(x, L_{b}^{-}, t\right)-\tilde{Z}^{n}(t)\right) \quad \text { and }\left.\quad \partial_{x} \tilde{\lambda}^{n}\right|_{x=L}=0
\end{aligned}
$$

with $C=\rho_{0} / M, C_{1}=\gamma /\left(M L_{i}\right), C_{2}=\left(R_{c}+R_{i}+n_{q} V_{T} / I_{\text {sat }}\right) / L_{i}$. By subtracting the $\tilde{Z}$-equation from the $\eta$-equation, we obtain:

$$
\frac{\left(\eta^{n+1}-\tilde{Z}^{n+1}\right)}{\Delta t}=\frac{\left(\eta^{n}-\tilde{Z}^{n}\right)}{\Delta t}-\tilde{W}^{n+1}-\nabla \cdot\left(H \nabla \tilde{\phi}^{n+1}\right) .
$$

Given that $\eta^{n}-\tilde{Z}^{n}=0$ and $\tilde{W}^{n}+\nabla \cdot\left(H \nabla \tilde{\phi}^{n}\right)=0$, both imposed at time level $n=0$ initially, ensuring that $\eta^{n+1}-\tilde{Z}^{n+1}=0$ implies that we have to show that

$$
\tilde{W}^{n+1}+\nabla \cdot\left(H \nabla \tilde{\phi}^{n+1}\right)=0
$$

Using the equation for $\tilde{W}^{n+1}$ and operating $\boldsymbol{\nabla} \cdot(H(x)(\cdot))$ on equation $(29 \mathrm{~b})$ for $\tilde{\phi}^{n+1}$, the consistency condition is seen to be $(29 \mathrm{~h})$. Hence, $(29)$ is consistent. 

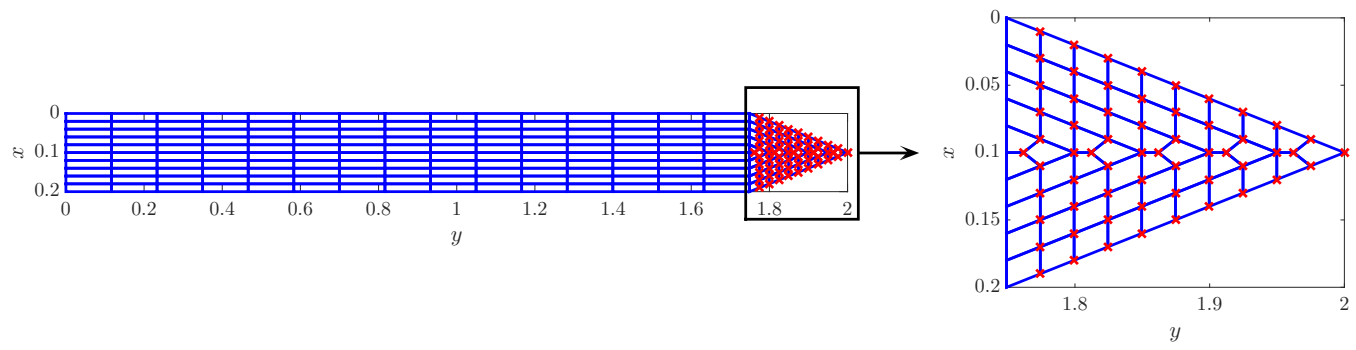

Figure 11. Computational mesh for $N_{x}=10, N_{y}=15$. The mesh structure in the contraction can be seen in the magnified right-hand-side plot, where the nodes in the contraction are denoted with a red $\times$ symbol.

\subsection{Space finite-element discretisation of the linearised system}

The model (27) is discretised in a few steps. The first step is to multiply the field equations in (27) by $C^{0}$-test functions, integrate over space and by parts. The second step is to expand the fields using (special) $C^{0}$-continuous and compact finite-element basis functions. We will use standard linear and compact Galerkin basis and test functions, which are unity at their home node and zero at neighbouring nodes of the elements connected to the home node. The result is a space-discrete system which will be revealed to be only consistent for certain special choices of the function spaces and expansions. Vice versa, we can first discretise time in a consistent manner such that again the equations remain consistent as we showed already. Finally, by either discretising the space-discrete system properly in time or the proper time discrete system in space, we obtain an internally consistent overall space-time discretisation fit for numerical implementation.

The mesh is assembled by using uniform quadrilateral elements up to the entrance of the contraction, with $N_{x}$ elements in the $x$-direction and $N_{y}$ elements in the $y$ direction, i.e. in the uniform section of the domain the mesh comprises $N_{x} N_{y}$ elements and $\left(N_{x}+1\right)\left(N_{y}+1\right)$ nodes. In the contraction the mesh is still formed by quadrilateral elements, but now nodes are only aligned in every other line. A sample mesh is shown in Fig. 11. The tessellation of the contraction region increases the total number of elements by $N_{x}\left(N_{x}+1\right) / 2$ and the number of nodes by $N_{x}\left(N_{x}+2\right) / 2$. It is thus clear that the way the mesh is constructed provides a restriction in the choice of $N_{x}$, which needs to be even. The total number of elements is $N_{e l}=N_{x} N_{y}+N_{x}\left(N_{x}+1\right) / 2$ and the total numbers of nodes is $N_{n}=\left(N_{x}+1\right)\left(N_{y}+1\right)+N_{x}\left(N_{x}+2\right) / 2$. The rest-state waterline is such that it is aligned with one of the nodal lines parallel to the $x$-direction.

The nodes in the entire mesh are denoted by $k, l=1,2, \ldots, N_{n}$ and the $N_{n}-N_{p}+1$ nodes under the buoy are in the ordered case denoted by $\tilde{k}, \tilde{l}=N_{p}, \ldots, N_{n}$. The latter include the nodes on the waterline, which in the current linearised case lies on the line $y=L_{b}$; the $N_{b}$ nodes on the waterline are a subset thereof, in the ordered case denoted by $\tilde{b}=N_{p}, \ldots, N_{p}+N_{b}-1$. When the $N_{b}$ nodes on the waterline are excluded in the latter we use index $\hat{k}$. We multiply both wave equations (27b)- 
(27c) by the $C^{0}$-test function $\varphi_{k}(x, y)$ and the elliptic equation (27h) as well as the constraint (27a) by $\varphi_{\hat{k}}(x, y)$ to obtain the weak forms after integration by parts, upon using the Neumann/Dirichlet conditions at $y=0$ and $x=l_{y}(x)$ for $x \in\left[0, L_{x}\right]$. Also, $\lambda_{h}(x, y, t)=\lambda_{\hat{k}}(t) \varphi_{\hat{k}}(x, y)+\lambda_{\tilde{b}}(t) \hat{\varphi}_{\tilde{b}}(x, y)$, with $\hat{\varphi}_{\tilde{b}}$ being the part of the test function $\varphi_{\tilde{b}} \geq L_{b}$ under the buoy and $\lambda_{\tilde{b}}=g\left(\eta_{\tilde{b}}-\tilde{Z}\right)$. Unfortunately, the consistency required cannot be shown for this choice of test function $\hat{\varphi}_{\tilde{b}}$ at the waterline. We therefore made some adjustments: we take $\lambda_{h}(x, y, t)=\lambda_{\tilde{k}}(t) \varphi_{\tilde{k}}(x, y)$ to be the normal test function spanning across the waterline with $\tilde{k}, \tilde{l}=N_{p}, \ldots, N_{n}$ and thus smooth out the Heaviside function to allow inclusion of the full basis function $\varphi_{\tilde{b}}$ at the waterline $y=L_{b}$. Hence, the Heaviside function $\Theta\left(y-L_{b}\right)$ is removed. This changes de facto only the vector and matrix definitions of $\tilde{S}_{\tilde{k} \tilde{l}}, \tilde{Q}_{\tilde{b}}$ and $N_{k \tilde{b}}$ below. The corresponding finite-element discretisation then becomes

$$
\begin{aligned}
N_{\tilde{k} l}^{T}\left(\eta_{l}-1_{l} \tilde{Z}\right) & =0 \\
M_{k l} \dot{\phi}_{l} & =-g M_{k l} \eta_{l}-N_{k \hat{l}} \lambda_{\hat{l}}-N_{k \tilde{b}} \lambda_{\tilde{b}} \\
M_{k l} \dot{\eta}_{l} & =S_{k l} \phi_{l}+T_{k} \dot{R} \\
\dot{\tilde{Z}} & =\tilde{W} \\
\dot{\tilde{W}} & =C \tilde{Q}_{\hat{l}} \lambda_{\hat{l}}+C \tilde{Q}_{\tilde{b}} \lambda_{\tilde{b}}-C_{1} G(\bar{Z})\left(\tilde{P}_{Q}+\gamma G(\bar{Z}) \tilde{Z}\right) \\
\dot{\tilde{Q}} & =\frac{\left(\tilde{P}_{Q}+\gamma G(\bar{Z}) \tilde{Z}\right)}{L_{i}} \\
\dot{\tilde{P}}_{Q} & =-C_{2}\left(\tilde{P}_{Q}+\gamma G(\bar{Z}) \tilde{Z}\right) \\
\left(\tilde{S}_{\tilde{k} \hat{l}}+C \tilde{Q}_{\tilde{k}} \tilde{Q}_{\hat{l}}\right) \lambda_{\hat{l}} & =-g S_{\tilde{k} l} \eta_{l}-C \tilde{Q}_{\tilde{k}} \tilde{Q}_{\tilde{b}} \lambda_{\tilde{b}}-\tilde{S}_{\tilde{k} \tilde{b}} \lambda_{\tilde{b}}+C_{1} \tilde{Q}_{\tilde{k}} G(\bar{Z})\left(\tilde{P}_{Q}+\gamma G(\bar{Z}) \tilde{Z}\right)
\end{aligned}
$$

with several mass and "Laplace" matrices defined by

$$
\begin{aligned}
M_{k l} & =\int_{0}^{L_{x}} \int_{0}^{l_{y}(x)} \varphi_{k}(x, y) \varphi_{l}(x, y) \mathrm{d} x \mathrm{~d} y, \\
S_{k l} & =\int_{0}^{L_{x}} \int_{0}^{l_{y}(x)} H(y) \boldsymbol{\nabla} \varphi_{k}(x, y) \cdot \nabla \varphi_{l}(x, y) \mathrm{d} x \mathrm{~d} y, \\
\tilde{S}_{\tilde{k} \tilde{l}} & =\int_{0}^{L_{x}} \int_{0}^{l_{y}(x)} H(y) \nabla \varphi_{\tilde{k}}(x, y) \cdot \nabla \varphi_{\tilde{l}}(x, y) \mathrm{d} x \mathrm{~d} y, \\
\tilde{Q}_{\tilde{k}} & =\int_{0}^{L_{x}} \int_{0}^{l_{y}(x)} \varphi_{\tilde{k}}(x, y) \mathrm{d} x \mathrm{~d} y, \\
N_{k \tilde{l}} & =\int_{0}^{L_{x}} \int_{0}^{l_{y}(x)} \varphi_{k}(x, y) \varphi_{\tilde{l}}(x, y) \mathrm{d} x \mathrm{~d} y, \\
T_{k} & =\int_{0}^{L_{x}} H(0) \varphi_{k}(0, y) \mathrm{d} y .
\end{aligned}
$$

Nonzero contributions only exist for the tilded matrices and vectors for certain index ranges. 
The key consistency check is to ensure that the first seven equations in (32) are consistent with the last seven equations in (32). Consider the first seven equations. Take the time derivative of the primary constraint and eliminate the time derivatives by using two of the other seven equations, to obtain the secondary constraint

$$
N_{\tilde{k} l}^{T}\left(M_{l k}^{-1}\left(S_{k m} \phi_{m}+T_{k} \dot{R}\right)-1_{l} \tilde{W}\right)=0 .
$$

Now take the time derivative of this secondary constraint above and again eliminate the time derivatives by using two different equations of these seven equations, to obtain the consistency equation

$$
\begin{gathered}
\left(N_{\tilde{k} l}^{T} M_{l k}^{-1} S_{k m} M_{m n}^{-1} N_{n \hat{l}}+C N_{\tilde{k} l}^{T} 1_{l} \tilde{Q}_{\hat{l}}\right) \lambda_{\hat{l}}=-g N_{\tilde{k} l}^{T} M_{l k}^{-1} S_{k m} \eta_{m} \\
-\left(N_{\tilde{k} l}^{T} M_{l k}^{-1} S_{k m} M_{m n}^{-1} N_{n \tilde{b}}+C N_{\tilde{k} l}^{T} 1_{l} \tilde{Q}_{\tilde{b}}\right) \lambda_{\tilde{b}} \\
\left.+N_{\tilde{k} l}^{T} M_{l k}^{-1} T_{k} \ddot{R}+C_{1} N_{\tilde{k} l}^{T} 1_{l} G(\bar{Z})\left(\tilde{P}_{Q}+\gamma G(\bar{Z}) \tilde{Z}\right)\right) .
\end{gathered}
$$

This consistency equation matches the last equation (32h) if and only if the following relations hold

$$
\begin{aligned}
\tilde{S}_{\tilde{k} \hat{l}} & =N_{\tilde{k} l}^{T} M_{l k}^{-1} S_{k m} M_{m n}^{-1} N_{n \hat{l}} \\
S_{\tilde{k} m} & =N_{\tilde{k} l}^{T} M_{l k}^{-1} S_{k m} \\
\tilde{S}_{\tilde{k} \tilde{b}} & =N_{\tilde{k} l}^{T} M_{l k}^{-1} S_{k m} M_{m n}^{-1} N_{n \tilde{b}} \\
\tilde{Q}_{\tilde{k}} & =N_{\tilde{k} l}^{T} 1_{l} \\
N_{\tilde{k} l}^{T} M_{l k}^{-1} T_{k} & =0 .
\end{aligned}
$$

These relations have been verified to hold up to machine precision. To date, we have not been able to verify these relations analytically. Finally, we find the consistent space-time discretisation by logically combining the time-discrete and space-discrete approaches derived in (29) and (32).

\subsection{Numerical results}

We set up a numerical code which simulates the full system as it evolves in time, including the generation/propagation of waves, their impact on the wave-energy buoy, the response of the buoy and the power output. The numerical results presented next have been obtained using a mesh resolution of $N_{x}=10, N_{y}=50$, i.e. the total number of elements in the calculations is $N_{e l}=555$ and the total number of nodes is $N_{n}=621$. The time step used is $\Delta t=0.0028$. At the start of the simulation the system is at rest and the water depth in the main wave tank is $H_{0}=0.1 \mathrm{~m}$. For $t>0$ waves are generated from the left wall of the tank by a piston wavemaker that follows a period motion in time according to $R(t)=\frac{A}{\omega}(1-\cos (\omega t))$, with amplitude $A=0.0653 \mathrm{~m}$ and frequency $\omega=\frac{6 \pi}{L_{y}} \sqrt{g H_{0}}=9.3348$ (which corresponds to a physical frequency of $\omega / 2 \pi=1.4857$ $\mathrm{Hz}$ ). Therefore on the left wall $\partial_{x} \tilde{\phi}=\dot{R}(t)=A \sin (\omega t)$. The response of the buoy is 

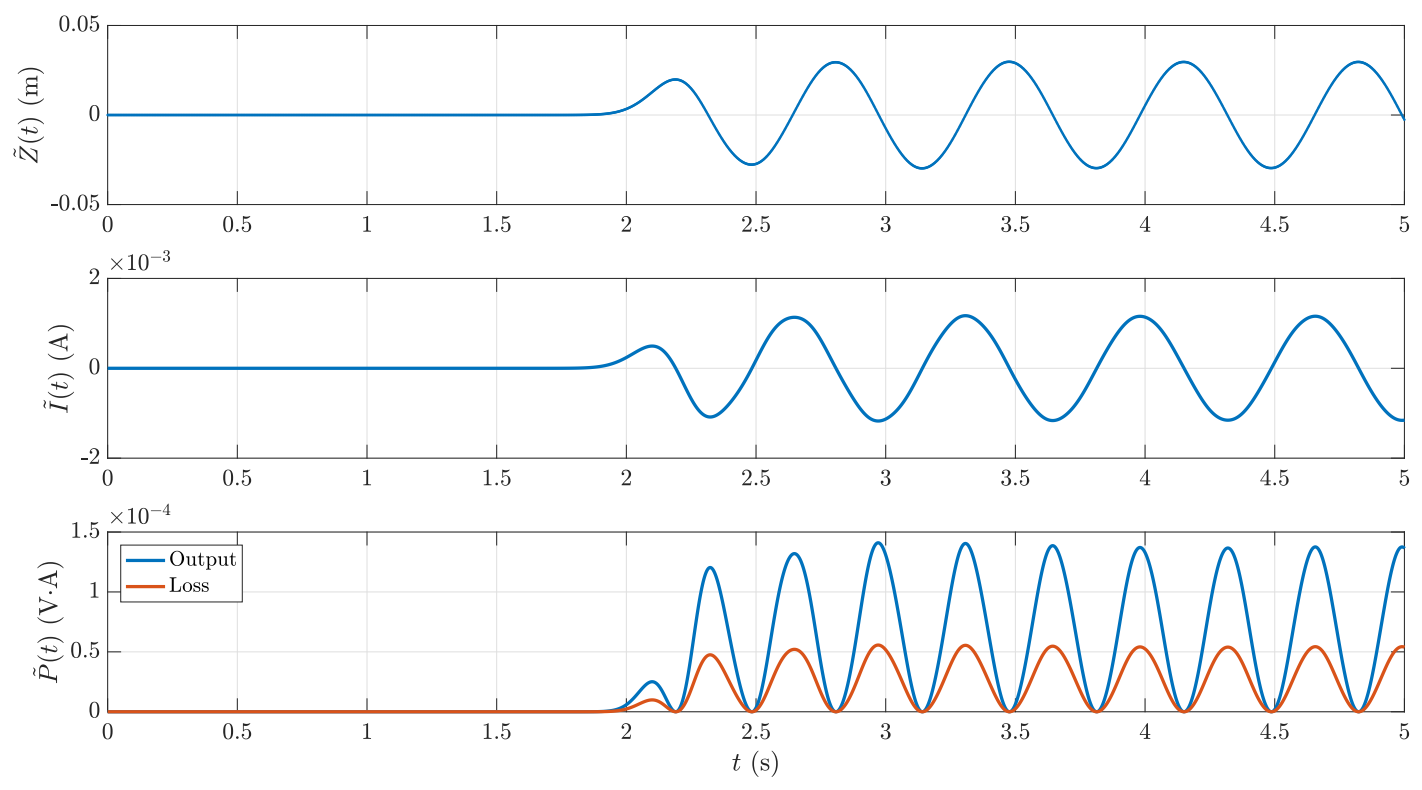

Figure 12. Vertical displacement of the buoy $\tilde{Z}(t)$ in meters $m$ (top panel), current $\tilde{I}(t)$ in amperes A (middle panel), and total power $\tilde{P}(t)$ in watts or volt times ampere $V \cdot A$ (bottom panel) generated by the LEDs (blue) or lost in the circuit (orange).

illustrated in the top panel of figure 12, while the current in the circuit can be seen in the middle panel and the power generated in the bottom panel; with blue line we show the power output at the LEDs, i.e. $\tilde{P}_{s}(t)=\tilde{I}(t) \tilde{V}_{s}(t)=n_{q} V_{T} / I_{\text {sat }} \tilde{I}^{2}(t)$, and with orange line the power lost due to the resistance in the circuit, i.e. $\tilde{P}_{r}(t)=\tilde{I}(t) \tilde{V}_{r}(t)=\left(R_{c}+R_{i}\right) \tilde{I}^{2}(t)$. Two snapshots of the numerically computed wave height and the position of the buoy in the contraction are demonstrated in figure 13.

\section{Summary and discussion}

In summary, we have reported in detail on the creation of the bore-soliton-splash, explored preliminary modelling of this hydrodynamic splash, and showed how it inspired a novel wave-energy device. We will next provide further context of our work.

Relation to rogue waves at sea: The bore-soliton-splash is a nonlinear waveresonance phenomenon in which a series of travelling solitons reflect in a V-shaped contraction leading to a tenfold resonant amplification of the initial main wave height. Once created in 2010, the phenomenon caught attention of the rogue-wave community. Rogue waves are extreme and rare waves, generally but not exclusively sea waves, at least twice as high as the wave height of the ambient sea. While the original boresoliton-splash was engineered, it relates to several rogue-wave phenomena at sea, far from and near the coastline. Rogue waves can have several causes and emerge in different situations: rogue waves emergence involves crossing seas, either due to seas 


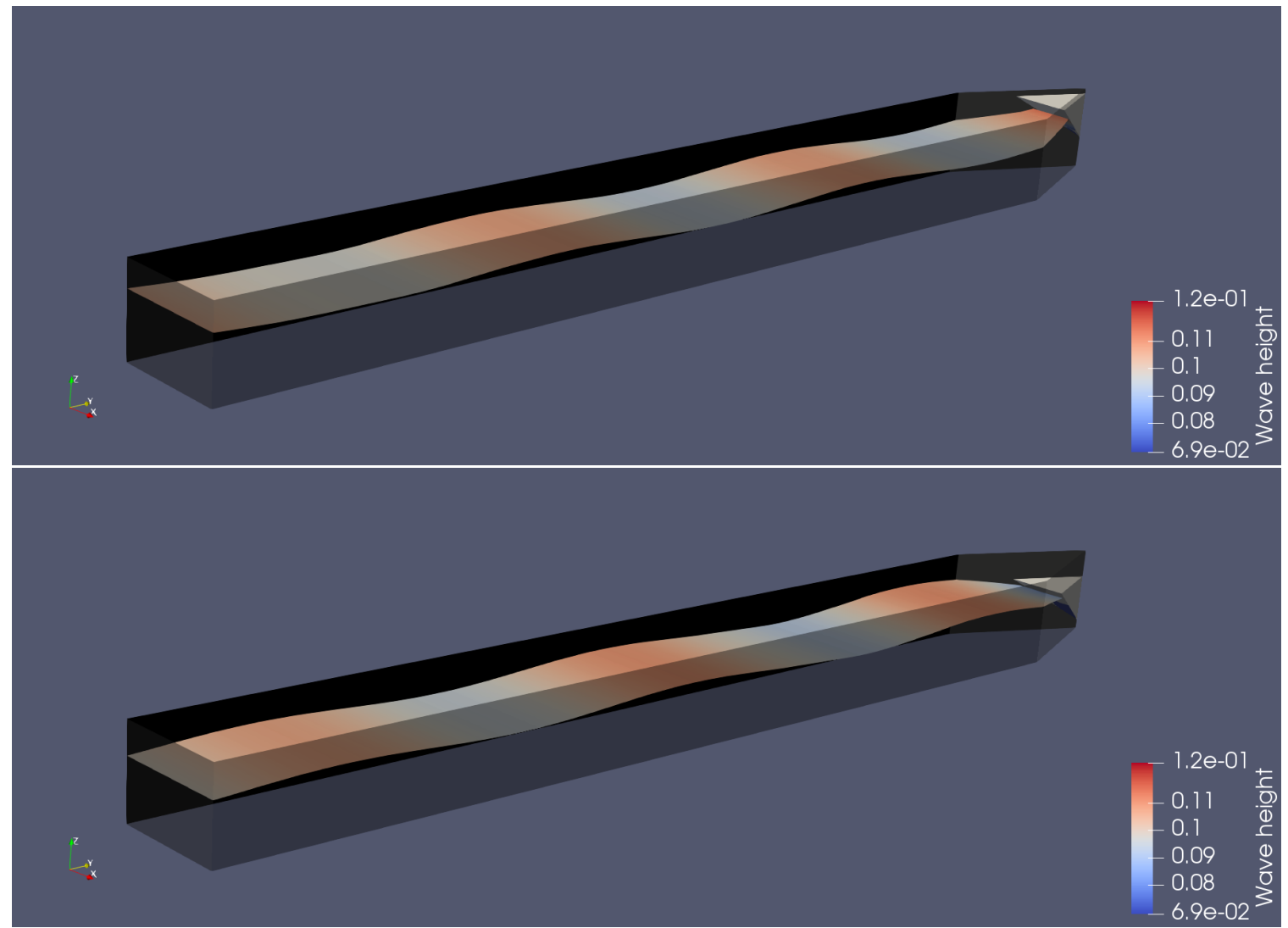

Figure 13. Snapshots from the simulation in a wave tank with V-shaped contraction and a wave-energy buoy in the corner of the contraction. The surface shown is the numerically computed wave height $h(x, y, t)=H(x, y)+\eta(x, y, t)$ (in meters $\mathrm{m}$ ) with $H_{0}=0.1$.

with two main directions, e.g. high seas generated by two hurricanes, or one hurricane changing direction. More rare are seas with waves and swell from three different main directions. Our V-shaped contraction walls can therefore be re-interpreted as virtual walls concerning two (virtual) waves travelling under two angles $\pm \varphi$ from the main wave's direction with two (virtual) planes of no-normal flow leading to a converging point. One difference is that the virtual case supports wave propagation with one splash at one space-time point, while our engineered set-up with solid walls necessarily leads to reflections.

Given this planar analogue, a simplified model with crossing seas is the KadomtsevPetviashvili (KP) equation [27, 31] for "nearly" uni- or $x$-directional waves with a weak, lateral $y$-structure. The KP equation allows exact web-soliton solutions for two interacting solitary waves colliding under an angle $[1,31]$. Two-soliton interactions on a plane relate to one soliton travelling along a solid wall and encountering a wall that turns $\varphi$ degrees inwards. Alternatively, this slanted wall can be seen as a virtual wall after two solitons in two channels under a slight angle encounter one another at the wall's convergence. For the optimal angle, two incoming solitons with an amplitude $A$ have 


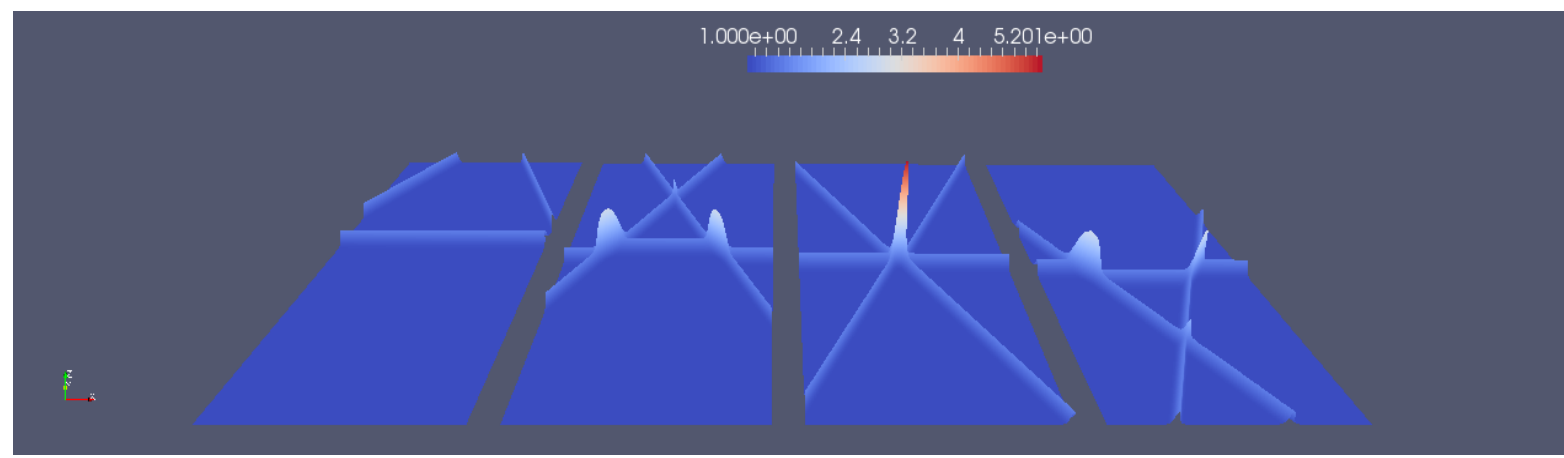

Figure 14. Three-soliton KP-interaction, each of amplitude $A=0.5$, leading to amplification of $8.6 \mathrm{~A}$. Interaction of three solitons (left panel) first leads to two twowave interactions, with a maximum amplification at one space-time point (third panel), whereafter the waves continue (right panel). Courtesy: Chloe Baker and Dr. Floriane Gidel [3, 22], using numerical coefficients provided by Prof. Yuji Kodama.

a maximum amplification $4 A$. Ablowitz and Curtis [1] found maximum amplification $3.9 A$ in simulations of the higher-order and bidirectional Benney-Luke equations, seeded with optimal KP-web-solitons as asymptotic solution. Gidel et al. [21] reached a maximum of $3.6 \mathrm{~A}$ in bidirectional Benney-Luke simulations of a soliton-wall collision, a more demanding calculation than a KP-web-soliton calculation, because waves require a long time to grow. We have exactly calculated a soliton-splash three-wave and planar analogue based on web-solitons [3, 22]. To date, for three colliding solitons each of amplitude $A$ maximum amplification is about 8.6A, as displayed in Fig. 14. Further work is required to determine, potentially analytically, for which angle maximum amplification occurs and whether it is particular to the KP-equation or also survives in more realistic water-wave models, i.e. Benney-Luke or potential-flow water-wave equations.

Tohoku Tsunami 2011: Coastal rogue waves are also related to our engineered and enclosed bore-soliton-splash and can arise due to topographical and geometrical amplification effects [41]. Destructive examples of coastal rogue waves were observed during the Tohoku Tsunami of 2011, cf. Lekkas et al. [34]. When a tsunami is aligned along an estuary and inland valley, it can lead to extremely large wave run-up, both in horizontal and vertical directions, potentially leading to destruction of villages high above sea level. The highest tsunami run-up for the Tohoku Tsunami was observed in Onagawa Bay, Miyagi Prefect, Japan, where an incoming tsunami with an estimated wave height of $7.6-8.5 \mathrm{~m}$ led to a vertical run-up of $42 \mathrm{~m}$ in a valley (cf. [34], their Figs. 2 and 3 and Table 1 ). The associated abnormality index was circa $A I=5.25$. Run-up was observed to be highest in narrow valleys with a high slope angle and the geographical set-up for these high-run-up cases is similar to the channels we used for our splash, but with vertical channel walls replaced by topographic valley walls with a slanted, contracting V-shape.

Further modelling of the bore-soliton-splash: We reported (bore-)soliton-splashes including one with smooth solitary waves in which nonlinearity and dispersion are 


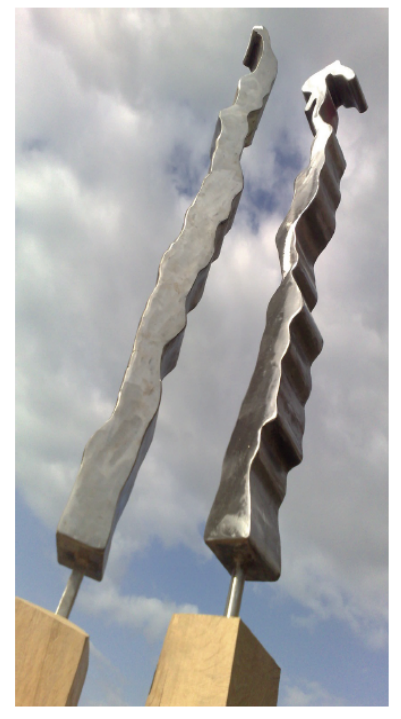

Figure 15. The steel-soliton splash is an artistic rendering in stainless steel of two snapshots of the bore-soliton-splash of Case 8.

balanced without any wave breaking. The smooth soliton-splash of Case 9 was successfully simulated using a compatible, geometric finite-element discretisation of a Benney-Luke model, a bidirectional simplification of the classic potential-flow model for water waves. Case 8 for the maximum bore-soliton-splash was not simulated correctly by the Benney-Luke model. Due to the lack of wave breaking, the Case 8 simulation deviated significantly from reality in that the observed resonant interaction was absent. Modelling the bore-soliton splash will either require a full potential-flow model with localised and parameterised wave-breaking or the use of models with actual, localised wave breaking while maintaining good dispersion properties. Single-phase or two-phase mixture-theory models, including ones with a Van-der-Waals-type equation of state, may be good candidates $[23,47]$.

Alternatively, we have explored use of Smoothed Particle Hydrodynamics (SPH)P; such a numerical method [24] can handle wave breaking and jet collapse into bubbles and droplets, and can be used to simulate the bore-soliton-splash of Case 8. SPH lies at the other end of the spectrum of numerical techniques, compared to the geometric numerical techniques used by us. To date, SPH does, however, turn out to be too dissipative. It requires too many degrees of freedom and thus too much computational power to avoid detrimental numerical wave-amplitude dissipation. Consequently, dispersive wave propagation over longer distances remains relatively poor in SPH. In our attempts to date the splash amplitude simulated by SPH was too low, even though we had replaced wave propagation in the channel prior to the contraction by several laterally periodic slices, copies of one another, to significantly reduce computational resources, before waves in these slices were fed into the contraction.

$\boldsymbol{P}$ Courtesy of Dr. Martin Robinson [45] https://www.youtube.com/watch?v=PRnyc06db1M 
Optimisation of the wave-energy device: Our splash inspired the creation of a novel wave-energy device and we showed a working, experimental proof-of-principle model, but also developed and derived a fully nonlinear mathematical model of the combined water-wave dynamics, the wave-buoy and the magnetic-induction actuator. Essential ingredients of this comprehensive model have been captured in one variational principle to which we a posteriori added dissipative effects of the electrical circuit, coils of the actuator and LEDs used as the loads. The overall model was subsequently linearised and discretised using a finite-element method in space and time. This (linear) algebraic model is made fully compatible with the variational structure in the conservative and continuum limits. Its compatible discretisation was augmented with resistances of induction motor, electrical circuit and LED-loads. Preliminary simulations of the linear model showed promising results. Nonlinear modelling, optimisation and control of the wave-energy device require further exploration, and both the geometry of contraction and wave-buoy shape could be optimised for a given wave climate. We also aim to explore feedback control.

Steel soliton-splash artwork: We finish on an artistic note. Our bore-soliton-splash inspired an artwork, the steel-soliton splash [50], created by WZ. Snapshots of the video of Case 8 were first outlined as silhouettes, two of which formed the basis for a threedimensional artwork, scaled down by about a factor of three, and welded in stainless steel, see Fig. 15. In 2013, we donated the artwork to the Isaac Newton Institute of Mathematical Sciences in Cambridge, UK. This is follow-up research that grew out of EPSRC grant EP/L025388/1 for AK and OB.

\subsection{Acknowledgments}

Part of this work appeared before in [4], in Dutch, as photographs and a first simulation of Case 9 in [5], and as illustrative photographs in [17]. Preliminary versions of the wave-energy device and its modelling appeared in conference proceedings [28, 29]. The story of the steel-soliton splash, first presented in [49], can be found on the website of the Isaac Newton Institute of Mathematical Sciences in Cambridge, UK. It is a pleasure to acknowledge assistance of Dr Paul Steenson and Prof Steve Tobias in formulating the magnetic-induction generator.

Note that this is a non-peer reviewed preprint submitted to EarthArXiv.

\section{References}

[1] M.J. Ablowitz and C.W. Curtis 2013: Conservation laws and web-solutions for the Benney-Luke equation. Proc. Roy. Soc. A 469, 20120690.

[2] B. Akers and O. Bokhove 2008: Hydraulic flow through a contraction: multiple steady states. Phys. Fluids 20, 056601.

[3] C. Baker 2017: Making a splash with solitons. Master-Math Thesis (supervised by OB), School of Mathematics, University of Leeds, $75 \mathrm{pp}$.

[4] O. Bokhove, E. Gagarina, W. Zweers and A. Thornton 2011: Bore Soliton Splash -van spektakel tot oceaangolf? Ned. Tijdschrift voor Natuurkunde 77/12, popular article in Dutch, 446-450. 
[5] O. Bokhove and A. Kalogirou 2016: Variational Water Wave Modelling: from Continuum to Experiment. Lecture Notes on the Theory of Water Waves Edited by: Bridges, Groves and Nicholls, London Mathematical Society Lecture Notes Series 426, 226-259.

[6] O. Bokhove, A. Kalogirou, D. Henry and G. Thomas 2019: A novel rogue-wave-energy device with wave amplification and induction actuator. Paper for European Wave and Tidal Energy Conference 2019, Napels. 10 pp. https://ewtec.org/conferences/ewtec-2019/

[7] H. Bredmose, D.H. Peregrine and G.N. Bullock 2009: Violent breaking wave impacts: Part 2. modelling the effect of air. J. Fluid Mech. 641, 389-430.

[8] L. Cavaleri, L. Bertotti, L. Torrisi, E. Bitner-Gregersen, M. Serio, M. Onorato 2012: Rogue waves in crossing seas: the Louis Majesty accident. J. Geophys. Res-Oceans 117, C00J10.

[9] L. Chen 2016: Maxwell's equations. https://www.math.uci.edu/ chenlong/226/MaxwellEqns. pdf

[10] C. Cotter and O. Bokhove 2010: Variational water-wave model with accurate dispersion and vertical vorticity. J. Eng. Math. 67, 33-54.

[11] G. Donoso, C.L. Ladera and P. Martin 2010: Magnetically coupled magnet-spring oscillators. European J. Physics 31, 433-452.

[12] G. Donoso, C.L. Ladera and P. Martin 2009: Magnet fall inside a conductive pipe. European J. Physics 310, 855-869.

[13] P.G. Drazin and R.S. Johnson 1989: Solitons: an introduction. Cambridge: Cambridge University Press.

[14] K. Dysthe, H.E. Krogstard and P. Muller 2008: Oceanic rogue waves. Ann. Rev. Fluid Mech. 40, $287-310$.

[15] A.F.O. Falcäo 2010: Wave energy utilization: A review of the technologies. Renewable and Sustainable Energy Reviews 14, 899-918.

[16] Faulkner 2001: Rogue waves: defining their characteristics for marine design. Rogue Waves 2000, Ifremer, France.

[17] E. Gagarina, J.J.W. van der Vegt and O. Bokhove 2013: Horizontal Circulation and Jumps in Hamiltonian Water Wave Model. Nonlinear Processes in Geophysics 20, 483-500.

[18] E. Gagarina, V. R. Ambati, J.J.W. van der Vegt and O. Bokhove 2014: Variational space-time (dis)continuous Galerkin method for nonlinear free surface water waves. J. Comput. Phys. 275, 459-483.

[19] E. Gagarina, V.R. Ambati, S. Nurijanyan, J.J.W. van der Vegt and O. Bokhove 2016: On variational and symplectic time integrators for Hamiltonian systems. J. Comput. Phys. 306, 370-389.

[20] K.F. Gieras, A. Kucharski and J. Pechowski 2017: Peformance characteristics of a shake flashlight. IEEE, 978-1-5090-4281-4/1 7/.

[21] F. Gidel, O. Bokhove and A. Kalgirou 2017: Variational modelling of extreme waves through oblique interaction of solitary waves: application to Mach reflection. Nonlinear Proc. Geophys. 24, 43-60.

[22] F. Gidel 2018: Variational water-wave models and pyramidal freak waves. PhD Thesis, University of Leeds, UK.

[23] F. Golay, M. Ersoy, L. Yushchenko and D. Sous 2015: Block-based adaptive mesh refinement scheme using numerical density of entropy production for three-dimensional two-fluid flows. Int. J. Comp. Fluid Dynamics 29, 67-81.

[24] M. Gómez-Gesteira, B.D. Rogers, R.A. Dalrymple and A.J.C. Crespo 2010: State-of-the-art of classical SPH for free-surface flows. J. Hydraulic Research 48, 6-27. doi : 10.3826/jhr . 2010.0012

[25] R.A. Howard, Y. Xiao, and S.D. Pekarek 2013: Modeling and design of air-core tubular electric devices. IEEE Trans Energy Conversion 28, 0885-8969.

[26] J.D. Jackson 1975: Classical Electrodynamics. Wiley, New York.

[27] B.B. Kadomtsev and V.I. Petviashvili 1970: On the stability of solitary waves in weakly dispersive media. Sov. Phys. Dokl. 15, 539-541. 
[28] A. Kalogirou and O. Bokhove 2016: Mathematical and numerical modelling of wave impact on wave-energy buoys. Proc. ASME 2016 35th Int. Conf. on Ocean, Offshore and Arctic Eng. OMAE . doi: 10.1115/OMAE2016-54937

[29] A. Kalogirou, O. Bokhove, D. Ham 2017: Modelling of nonlinear wave-buoy dynamics using constrained variational methods. Proc. Int. Conf. on Offshore Mechanics and Arctic Engineering - OMAE. doi:10.1115/OMAE2017-61966

[30] C. Khariff, E. Pelinovsky, A. Slunyaev, 2009: Rogue Waves in the Ocean. Advances in geophysical and environmental mechanics and mathematics. Springer. 216 pp.

[31] Y. Kodama 2010: KP solitons in shallow water. Journal of Physics A: Mathematical and Theoretical 43, 434004

[32] D. J. Korteweg and G. de Vries, 1895: On the change of form of long waves advancing in a rectangular canal, and on a new type of long stationary waves. Philosophical Magazine, 39 (240), 422-443.

[33] B. Leimkuhler and S. Reich 2010: Simulating Hamiltonian Dynamics. Cambridge University Press. 379 pp.

[34] E. Lekkas, E. Andreadakis, I. Kostaki and E. Kapourani 2011: Critical Factors for Run-up and Impact of the Tohoku earthquake Tsunami. Int. J. Geosci. 2, 310-317. See also: https: //www. newyorker.com/news/news-desk/the-fall-and-rise-of-onagawa

[35] P. Lorrain and D. Corson 1970: Electromagnetic fields and waves. Freeman, New York.

[36] J. Luke 1967: A variational principle for a fluid with a free surface. J. Fluid Mech. 27, 395-397.

[37] F. Madhi and R.W. Yeung 2018: On survivability of asymmetric wave-energy converters in extreme waves. Renewable Energy 119, 891-909.

[38] K. McCarthy, M. Bash and S. Pekarek 2008 Design of an air-core linear generator drive for energy harvest applications. IEEE 978-1-4244-1874-9/08/.

[39] P.A. Milewski and J.B. Keller, J.B. 1996: Three-dimensional water waves. Studies in Applied Mathematics 97, 149-166.

[40] http://web.mit.edu/6.013_book/www/chapter9/9.3.html

[41] I. Nikolkina and I. Didenkulova 2011: Rogue waves in 2006-2010. Nat. Hazards Earth Syst. Sci. 11, 2913-2924.

[42] M. Onerato, D. Proment, G. Clauss and M. Klein 2013: Rogue waves: from nonlinear Schrödinger breather solutions to seakeeping tests. Plos One 8, e54629.

[43] R.L. Pego, J.R. Quintero 1999: Two-dimensional solitary waves for a BenneyLuke equation. Physica D 132, 476-496.

[44] F. Rathgeber, D.A. Ham, L. Mitchell, M. Lange, F. Luporini, A.T.T. McRae, G. Bercea, G.R. Markall and P.H.J. Kelly 2016: Firedrake: automating the finite element method by composing abstractions. ACM TOMS Trans. Math. Softw. 43 1-27. https://www.firedrakeproject.org/

[45] M. Robinson, P. Cleary, J.J. Monaghan 2008: Analysis of mixing in a twin cam mixer using smoothed particle hydrodynamics. AIChE J. 54, 1987B198.

[46] D.H. Peregrine 2003: Water-wave impact on walls. Annu. Rev. Fluid Mech. 35, 23-43.

[47] T. Salwa 2018: On variational modelling of wave slamming by water waves. PhD Thesis. Submitted Sept. 2018. University of Leeds, UK.

[48] W. Zweers 2010: Youtube channel https://www.youtube.com/user/woutzweers; especially videos with titles "soliton splash 27 sep run 3.mp4", "soliton splash 27 sep run 6.mp4" and "Soliton splash opening O\&O plein Utwente" (of 30-09-2010) as well as "soliton splash" (of 2009-2010) and the portable soliton set-up (1-14-2011) "Portable Bore-Soliton-Splash".

[49] W. Zweers, V. Zwart and O. Bokhove 2013: Making waves: visualizing fluid flows. Proc. Bridges 2013: Mathematics, Music, Art, Architecture, Culture. Eds. George W. Hart and Reza Sarhangi. 515-518. ISBN 978-1-938664-06-9 http://archive.bridgesmathart.org/2013/ bridges 2013-515.html

[50] W. Zweers 2014: Steel-soliton splash. Artwork at Isaac Newton Institute of Mathematical sciences: https://www.newton.ac.uk/about/ 
art-artefacts/soliton and https://waterwaves2014.wordpress.com/2014/07/21/ onno-bokhove-donates-steel-soliton-splash-sculptures-to-newton-institute/

\section{Appendix A. Model of induction generator}

The induction motor modelled consists of a series of permanent cylindrical magnets attached to the top of the wave-buoy's vertical mast. These magnets move through a series of coils connected in a circuit to two (sets of) LEDs, the loads, placed in two parallel directions, with half of the LEDs placed in one direction and the other half in the other direction. The latter placement guarantees that always one (set of) LED(s) is lighting up under the alternating current. The first modelling step is to calculate the magnetic flux $\mathbf{B}$ induced in the series of coils. The second modelling step is to calculate the voltage and current across and through the coils using Ohm's law for the current density, electrical field and magnetic flux. This moving magnetic flux $\mathbf{B}$ induces a current $I=I(t)$ in the coils. The third modelling step is to calculate the magnetic force and, via Newton's law, the force on the buoy-mast-magnet system ${ }^{+}$.

First step: The magnetic flux of a magnet, a cylinder magnetised in the direction of its axis of symmetry, is identical to that of a solenoid of the same dimension with a current density $N^{\prime} i=\mu$, here $i$ is a fictitious current as opposed to the current $I$ we aim to model, with

$$
\mu=\mu_{0} m /(4 \pi)
$$

the product of $\mu_{0}=4 \pi \times 10^{-7} \mathrm{H} / \mathrm{m}$ the permeability of free space times the magnetic/magnet's dipole moment $m$ divided by $4 \pi$, and $N^{\prime}$ the number density of the turns per metre (Lorrain and Corson [35] pp. 393). We start with the magnetic flux generated by a current in a single coil solenoid, cf. [26, 35] (Jackson pp. 178 and Lorrain and Corson, pp. 319) and subsequently via integration along the magnet extend that to an entire solenoid with multiple turns equivalent to our magnet.

In spherical coordinates $(r, \varphi, \theta)$ and with the centre of the coil placed at the origin, the approximate expressions $[26,35]$ of the two nonzero components of the magnetic flux, for distances $r \gg A_{m}$ larger than the radius $A_{m}$ of the magnet, are given by the spherical-coordinate components $B_{r}=\frac{2 \mu \cos \theta}{r^{3}}, B_{\theta}=\frac{\mu \sin \theta}{r^{3}}$ of the magnetic flux $\mathbf{B}$, or rewritten in cylindrical coordinates $(\rho, \phi, z)$ for the radial cylindrical-coordinate component of the magnetic flux

$$
B_{\rho}=B_{r} \sin \theta+B_{\theta} \cos \theta=\frac{3 \mu z \rho}{\left(\rho^{2}+z^{2}\right)^{5 / 2}} .
$$

The magnetic field $\mathbf{B}$ is expressed in Teslas, $\mathrm{T}$, the permeability $\mu_{0}$ of free space in Henry's per metre, $\mathrm{H} / \mathrm{m}=\mathrm{Tm}^{2} / \mathrm{A}$, the dipole moment $m$ in Ampere-square-metre, $\mathrm{Am}^{2}$, and the radius $r$ in metres. The above holds for an infinitesimally short magnet and far away from the magnet, relative to the distance at which the magnetic flux

+ The model we formulate is based on a compilation and nonlinear extension of material in $[11,12,26,35]$ with the work of Donoso et al. [11, 12] concerning coupled mechanical-magnetic systems. 
is measured and relative to the length of the coils, cf. [12] (their (1) and (2)). The magnetic field induced by a magnet of finite length $L_{m}$ is considered next and reduces in the far field to the above expression.

The magnetic flux $\mathbf{B}$ is related to the magnetic field intensity $\mathbf{H}$ and magnetisation density $\mathbf{M}$ as follows (page 395 of [35])

$$
\mathbf{B}=\mu_{0}(\mathbf{H}+\mathbf{M}) .
$$

In the absence of a current density $\mathbf{J}$, the magnetic field density is irrotational such that $\mathbf{H}=-\boldsymbol{\nabla} \Psi$ outside the magnet, with magnetic scalar potential $\Psi$. Since $\boldsymbol{\nabla} \cdot \mathbf{B}=0$, we find from (A.3) that $\boldsymbol{\nabla} \cdot \mathbf{H}=-\boldsymbol{\nabla} \cdot \mathbf{M}$ and therefore that

$$
\nabla^{2} \Psi=-\frac{\rho_{m}}{\mu_{0}} \equiv \nabla \cdot \mathbf{M}
$$

with magnetic charge density $\rho_{m}$. The integral solution of (A.4) reads

$$
\Psi=\iiint_{V^{\prime}} \frac{\rho_{m}\left(\mathbf{r}^{\prime}\right)}{4 \pi \mu_{0}\left|\mathbf{r}-\mathbf{r}^{\prime}\right|} \mathrm{d} V^{\prime} .
$$

For a magnet uniformly magnetised in the $z$-direction, we have a magnetisation density $\mathbf{M}=M_{0} \hat{\mathbf{z}}$ or, alternatively, the magnetic density $\rho_{m}=0$ throughout the magnet except at the end surfaces where $\rho_{m}= \pm \mu_{0} M_{0} \delta\left(z^{\prime} \mp L_{m} / 2\right)$. The magnetic scalar potential $\Psi$ is given by the triple integral (A.5) over the length $L_{m}$ of the magnet of radius $A_{m}<a$ with $a$ the radius of the inductor's coils, cf. [35] (pp. 395) and [40]:

$$
\begin{array}{rl}
\Psi(\rho, z)=\frac{M_{0}}{4 \pi} \int_{0}^{2 \pi} \int_{0}^{A_{m}} & R\left(\frac{1}{\left(R^{2}+\rho^{2}-2 R \rho \cos \phi+\left(z-L_{m} / 2\right)^{2}\right)^{1 / 2}}\right. \\
& \left.-\frac{1}{\left(R^{2}+\rho^{2}-2 R \rho \cos \phi+\left(z+L_{m} / 2\right)^{2}\right)^{1 / 2}}\right) \mathrm{d} R \mathrm{~d} \phi
\end{array}
$$

in which, based on symmetry, it suffices to take $\tilde{\phi}=0$ with $x=\rho \cos \tilde{\phi}$ etc. such that $\left|\mathbf{r}-\mathbf{r}^{\prime}\right|^{2}=(R \cos \phi-\rho)^{2}+R^{2} \sin ^{2} \phi+\left(z-z^{\prime}\right)^{2}$. The expression of the relevant component of the magnetic field evaluated for a magnet of length $L_{m}$ and radius $A_{m}<a$ then becomes:

$$
\begin{aligned}
B_{\rho}(\rho, z)= & -\mu_{0} \frac{\partial \Psi_{\phi}}{\partial \rho} \\
= & \frac{\mu_{0} M_{0}}{4 \pi} \int_{0}^{2 \pi} \int_{0}^{A_{m}}\left(\frac{R(\rho-R \cos \phi)}{\left(R^{2}+\left(L_{m} / 2-z\right)^{2}+\rho^{2}-2 R \rho \cos \phi\right)^{3 / 2}}\right. \\
& \left.\quad-\frac{R(\rho-R \cos \phi)}{\left(R^{2}+\left(L_{m} / 2+z\right)^{2}+\rho^{2}-2 R \rho \cos \phi\right)^{3 / 2}}\right) \mathrm{d} R \mathrm{~d} \phi .
\end{aligned}
$$

Far away from the magnet, this radial component can be approximated by using Taylor 
Bore-soliton-splash $\&$ wave-energy device

expansions

$$
\begin{aligned}
B_{\rho}(\rho, z) \approx & \frac{\mu_{0} M_{0}}{4 \pi} \int_{0}^{2 \pi} \int_{0}^{A_{m}} \frac{R \rho}{\left(z^{2}+\rho^{2}\right)^{3 / 2}}\left(\frac{1}{\left(1+\left(-z L_{m}+L_{m}^{2} / 4\right) /\left(z^{2}+\rho^{2}\right)\right)^{3 / 2}}\right. \\
& \left.\quad-\frac{1}{\left(1+\left(z L_{m}+L_{m}^{2} / 4\right) /\left(z^{2}+\rho^{2}\right)\right)^{3 / 2}}\right) \mathrm{d} R \mathrm{~d} \phi \\
& \approx \frac{\mu_{0} M_{0}}{4 \pi} \int_{0}^{2 \pi} \int_{0}^{A_{m}} \frac{3 L_{m} z R \rho}{\left(z^{2}+\rho^{2}\right)^{5 / 2}} \mathrm{~d} R \mathrm{~d} \phi \\
& =\frac{\mu_{0} M_{0} \pi A_{m}^{2} L_{m}}{4 \pi} \frac{3 z \rho}{\left(z^{2}+\rho^{2}\right)^{5 / 2}},
\end{aligned}
$$

which equals (A.2) with (A.1) when we identify $m=M_{0} \pi A_{m}^{2} L_{m}$ as the magnetic/magnet's dipole moment with $\pi A_{m}^{2} L_{m}$ the volume taken up by the magnet. In (A.7), the magnet is placed in $z \in\left[-L_{m} / 2, L_{m} / 2\right]$, while in our wave-energy device the magnet resides between $z \in\left[Z(t)+H_{m}-L_{m} / 2, Z(t)+H_{m}+L_{m} / 2\right]$. Consequently, we need to adapt expression (A.7) as follows

$$
\begin{aligned}
\tilde{B}_{\rho}(\rho, z)= & \frac{\mu_{0} M_{0}}{4 \pi} \int_{0}^{2 \pi} \int_{0}^{A_{m}}\left(\frac{R(\rho-R \cos \phi)}{\left(R^{2}+\left(L_{m} / 2-z+Z+H_{m}\right)^{2}+\rho^{2}-2 R \rho \cos \phi\right)^{3 / 2}}\right. \\
& \left.-\frac{R(\rho-R \cos \phi)}{\left(R^{2}+\left(L_{m} / 2+z-Z-H_{m}\right)^{2}+\rho^{2}-2 R \rho \cos \phi\right)^{3 / 2}}\right) \mathrm{d} R \mathrm{~d} \phi \\
\approx & \frac{3 \mu \rho\left(z-Z-H_{m}\right)}{\left(\left(z-Z-H_{m}\right)^{2}+\rho^{2}\right)^{5 / 2}} .
\end{aligned}
$$

Second step: Placing the magnet in a moving range $z \in\left[Z(t)+H_{m}-L_{m} / 2, Z(t)+\right.$ $\left.H_{m}+L_{m} / 2\right]$ with length $H_{m}$ above the centre of mass $Z(t)$ and for fixed coils with $N$ turns and a radius $\rho=a$ over a coil length $L$ over a fixed range $z \in\left[\bar{Z}+\left(1+\alpha_{h}\right) H_{m}-\right.$ $L / 2, \bar{Z}+\left(1+\alpha_{h}\right) H_{m}+L / 2$ ] with $0<\alpha_{h}<1$, the expression (A.9a) can be integrated in $z$ over the coil to obtain the coil density (cf. [12] their (6)-(8))

$$
\begin{aligned}
& \epsilon_{1}(Z)=\frac{N}{L} \int_{\bar{Z}+\left(1+\alpha_{h}\right) H_{m}-L / 2}^{\bar{Z}+\left(1+\alpha_{h}\right) H_{m}+L / 2} \tilde{B}_{\rho}(\rho=a, z) \mathrm{d} z \\
& \epsilon_{1}(Z)=\frac{N}{L} \int_{-L / 2}^{L / 2} \tilde{B}_{\rho}\left(\rho=a, z=q+\bar{Z}+\left(1+\alpha_{h}\right) H_{m}\right) \mathrm{d} q \\
& =\frac{a \mu N}{L} G(Z) \\
& \approx \frac{N}{L} \int_{-L / 2}^{L / 2} \frac{3 \mu a\left(q+\bar{Z}+\alpha_{h} H_{m}-Z\right)}{\left(a^{2}+\left(q+\bar{Z}+\alpha_{h} H_{m}-Z\right)^{2}\right)^{5 / 2}} \mathrm{~d} q \\
& =\frac{a \mu N}{L}\left(\frac{1}{\left(a^{2}+\left(\bar{Z}+\alpha_{h} H_{m}-Z-L / 2\right)^{2}\right)^{3 / 2}}\right. \\
& \left.-\frac{1}{\left(a^{2}+\left(\bar{Z}+\alpha_{h} H_{m}-Z+L / 2\right)^{2}\right)^{3 / 2}}\right) \text {, }
\end{aligned}
$$



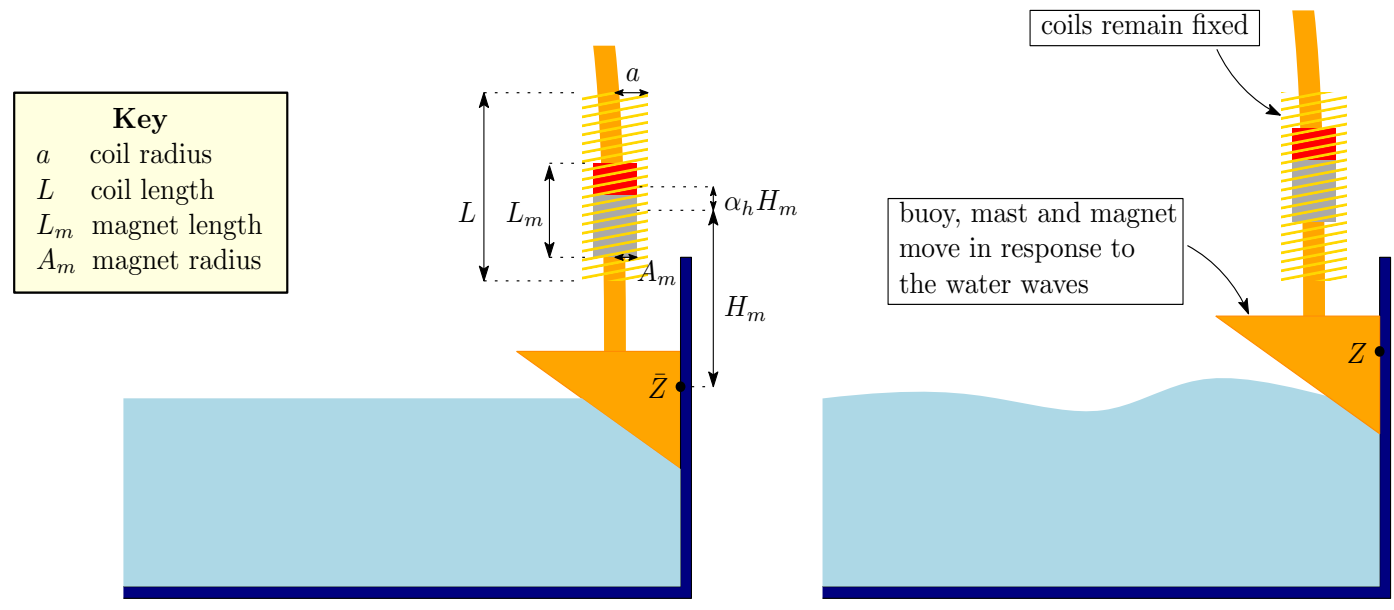

Figure A1. Sketch of the magnetic induction-motor set-up: on the left the system when the magnet passes its rest position and on the right away from the rest position.

where we have used that at the faces of the moving magnet

$L_{m} / 2-z+Z+H_{m}=L_{m} / 2-q-\bar{Z}-\alpha_{h} H_{m}+Z, \quad L_{m} / 2+z-Z-H_{m}=L_{m} / 2+q+\bar{Z}+\alpha_{h} H_{m}-Z$

and defined the function

$$
\begin{aligned}
G(Z)= & \frac{1}{\pi A_{m}^{2} L_{m} a} \int_{-L / 2}^{L / 2} \int_{0}^{2 \pi} \int_{0}^{A_{m}}\left(\frac{r(a-r \cos \phi)}{\left(r^{2}+\left(\frac{L_{m}}{2}-q-\bar{Z}-\alpha_{h} H_{m}+Z\right)^{2}+a^{2}-2 r a \cos \phi\right)^{3 / 2}}\right. \\
& \left.-\frac{r(a-r \cos \phi)}{\left(r^{2}+\left(\frac{L_{m}}{2}+q+\bar{Z}+\alpha_{h} H_{m}-Z\right)^{2}+a^{2}-2 r a \cos \phi\right)^{3 / 2}}\right) \mathrm{d} r \mathrm{~d} \phi \mathrm{d} q \quad(\text { A. } 10 \mathrm{f}) \\
\approx & \frac{1}{\left(a^{2}+\left(\bar{Z}+\alpha_{h} H_{m}-Z-L / 2\right)^{2}\right)^{3 / 2}}-\frac{1}{\left(a^{2}+\left(\bar{Z}+\alpha_{h} H_{m}-Z+L / 2\right)^{2}\right)^{3 / 2}},
\end{aligned}
$$

with the latter approximation holding in the far field, starting from (A.10d) and (A.9b). Note that only when $\alpha_{h}=0$ and the water and the buoy-mast-magnet system are at rest, the magnet sits in the middle of the coils. A sketch of the various coordinate systems is given in Fig. A1. A graph of $\gamma G(Z)$ versus $Z$ and its approximation shows that the two functions lie close together, cf. [6]

Ohm's law for a circuit subject to a magnetic flux, moving with a speed $\dot{Z}$ in the vertical, reads

$$
\mathbf{J}=\sigma(\mathbf{E}+\dot{Z} \hat{\mathbf{z}} \times \mathbf{B})
$$

with current density $\mathbf{J}$, electrical field $\mathbf{E}$, conductivity $\sigma>0$ for a conductor, and unit vector $\hat{\mathbf{z}}$ in the vertical. Integration of Ohm's law (A.11) around the coils at $\rho=a$ yields 
(extending $[12,9])$

$$
\begin{aligned}
\oint \frac{\mathbf{J}}{\sigma} \cdot \mathrm{d} \mathbf{l}=\frac{I}{\pi D^{2} / 4} \frac{N 2 \pi a}{\sigma} \equiv I R_{c} & =\int_{-L / 2}^{L / 2} \oint \mathbf{E}+\dot{Z} \hat{\mathbf{z}} \times \mathbf{B} \cdot \mathrm{d} \mathbf{l} \mathrm{d} q \\
& =-L_{i} \frac{\mathrm{d} I}{\mathrm{~d} t}+2 \pi a \epsilon_{1}(Z) \dot{Z} \\
& =-L_{i} \frac{\mathrm{d} I}{\mathrm{~d} t}+\frac{2 \pi a^{2} N \mu}{L} \dot{Z} G(Z) \\
& \equiv-L_{i} \frac{\mathrm{d} I}{\mathrm{~d} t}+V_{c}
\end{aligned}
$$

where $\mathrm{d} \mathbf{l}=a \mathrm{~d} \theta(-\sin \theta, \cos \theta, 0)^{T}$ and $\mathbf{B}=\tilde{B}_{\rho}(\cos \theta, \sin \theta, 0)^{T}$ with $\theta \in[0,2 \pi]$, inductance $L_{i}$ of the inductor and the voltage drop $V_{c}$. Note that the resistance of the coils is defined by $R_{c}=N(2 \pi a) /\left(\sigma \pi D^{2} / 4\right)$ and the current magnitude by $J=I /\left(\pi D^{2} / 4\right)$ with $D$ the cross-sectional diameter of the coil. Hence, the circuit equation becomes

$$
I\left(R_{c}+R_{i}\right)=-L_{i} \dot{I}+V_{c}+V_{s}(I)
$$

where we have added the resistance $V_{s}(I)$ (19) of the two (sets of) LEDs modelled using combined Shockley equations, placed in parallel, as well as the resistance $R_{i}$ of the remaining wires to and from these LEDs. It should be possible top add $I R_{i}$ and the Shockley voltage $V_{s}$ directly in Ohm's law but we simply added the two terms heuristically.

Faraday's induction law (chapters 7 and 8 [35]) used above follows from one of the Maxwell's equations, $\boldsymbol{\nabla} \times \mathbf{E}=-\partial_{t} \mathbf{B}$, for one circuit as

$$
\oint \mathbf{E} \cdot \mathrm{d} \mathbf{l}=-\frac{\mathrm{d}}{\mathrm{d} t} \iint_{S} \mathbf{B} \cdot \mathrm{d} \mathbf{a}=-\frac{\mathrm{d} \Phi}{\mathrm{d} t}=-L_{i} \dot{I},
$$

with induction $\Phi$, surface $S$ bounded by the path of the line integral and surface element da on the coils of the inductor. The magnetic induction inside a long solenoid is $B=\mu_{0}(N / L) I$ (cf. (8.59) in [35]) such that $\Phi=\mu_{0}(N / L) I \pi a^{2}$ (cf. (8.60) in [35]) and, hence, for $N$ windings $L_{i}=K \mu_{0}\left(N^{2} / L\right) \pi a^{2}$ (cf. (8.61) in [35]). For a long solenoid $K=1$ and for a short solenoid $K<1$ with $K=0.53$ for $a / L=1$ (Table 8-1 in [35]).

Third step: Faraday's expression for the magnetic force $\mathbf{F}$, the force on charged particles in the coils as used in the momentum equations of magneto-hydrodynamics, is

$$
\begin{aligned}
\mathbf{F} & =\iiint \mathbf{E}+\mathbf{J} \times \mathbf{B} \mathrm{d} V=\iiint \mathbf{E} \mathrm{d} V+I \oint \mathrm{d} \mathbf{l} \times \mathbf{B} \\
& =\frac{N}{L} \int_{0}^{\infty} \int_{0}^{2 \pi} \int_{-L / 2}^{L / 2} \rho I \delta(\rho-a) \tilde{B}_{\rho}(-\sin \theta, \cos \theta, 0)^{T} \times(\sin \theta, \cos \theta, 0)^{T} \mathrm{~d} \rho \mathrm{d} \theta \mathrm{d} q
\end{aligned}
$$

in which we have replaced the magnitude of $\mathbf{J}$ integrated across a string of coil with area $\Delta S$ by $I / \Delta S$ multiplied times $\Delta S \delta(\rho-a)$ to focus its averaged effect at $\rho=a$ exclusively. Hence, the vertical component $F=\mathbf{F} \cdot \hat{\mathbf{z}}$ of the force between the conducting 
coils and the magnet is

$$
F=\int_{-L / 2}^{L / 2} \oint I \mathrm{~d} \mathbf{l} \times \mathbf{B} \cdot \hat{\mathbf{z}} \mathrm{d} q=-\frac{2 \pi a^{2} N \mu I}{L} G(Z) .
$$

To facilitate further analysis, we momentarily simplify the hydrodynamic force on the buoy, used in the main text, by a mass-spring component with spring constant $k_{s}$. Combining this simplification with the vertical momentum equation of the simplified buoy-mast system, we then arrive at the following coupled mechanical and magnetical system

$$
\begin{aligned}
\dot{Q} & =I \\
L_{i} \dot{I} & =\gamma G(Z) \dot{Z}-\left(R_{c}+R_{i}\right) I-\operatorname{sign}(I) n_{q} V_{T} \ln \left(\frac{|I|}{I_{s a t}}+1\right) \\
\dot{Z} & =W \\
M \dot{W} & =-M g-k_{s} Z-\gamma G(Z) I
\end{aligned}
$$

with constant $\gamma \equiv 2 \pi a^{2} \mu N / L$ and underlined dissipative terms. When we ignore the self-induction term $L_{i} \dot{I}$ and the Shockley expression for the LEDs in (A.17), we note that $\left(R_{c}+R_{i}\right) I=\gamma G(Z) \dot{Z}$; elimination of $I$ then shows that the magnetic force in the vertical momentum equation (A.17d) acts as a (nonlinear) drag, proportional to $\dot{Z}$ or $W$, cf. the linear analogue in [12]. In the absence of the underlined, linear and nonlinear dissipative terms in (A.17), the system (A.17) should be conservative, which will be explored next. In this conservative limit, we first rewrite (A.17) as

$$
L_{i} \ddot{Q}-\gamma G(Z) \dot{Z}=0 \quad \text { and } \quad M \ddot{Z}+M g+k_{s} Z+\gamma G(Z) \dot{Q}=0 .
$$

The system (A.18) has a Lagrangian $L_{m m}$, defined in and satisfying the following variational principle

$$
\begin{aligned}
0 & =\delta \int_{0}^{T} \mathrm{~L}_{m m}(Z, \dot{Z}, Q, \dot{Q}) \mathrm{d} t \\
& \equiv \delta \int_{0}^{T} \frac{1}{2} M \dot{Z}^{2}+\frac{1}{2} L_{i} \dot{Q}^{2}-M g Z-\frac{1}{2} k_{s} Z^{2}+\gamma G(Z) Q \dot{Z} \mathrm{~d} t \\
& =\int_{0}^{T}-\left(M \ddot{Z}+M g+k_{s} Z+\gamma G(Z) \dot{Q}+\gamma G^{\prime}(Z) \dot{Z} Q-\gamma G^{\prime}(Z) \dot{Z} Q\right) \delta Z \\
& \quad+\left(-L_{i} \ddot{Q}+\gamma G(Z) \dot{Z}\right) \delta Q \mathrm{~d} t \\
& =\int_{0}^{T}-\left(M \ddot{Z}+M g+k_{s} Z+\gamma G(Z) \dot{Q}\right) \delta Z+\left(-L_{i} \ddot{Q}+\gamma G(Z) \dot{Z}\right) \delta Q \mathrm{~d} t
\end{aligned}
$$

for end-point conditions $\delta Q(0)=\delta Q(T)=\delta Z(0)=\delta Z(T)=0$. The equations of motion in (A.18) follow from the arbitrariness of the variations in the last expression of (A.19). To facilitate a Legendre transform, the variational principle (A.19) is rewritten 
as

$$
\begin{aligned}
0 & =\delta \int_{0}^{T} \mathrm{~L}_{m m}(Z, \dot{Z}, Q, \dot{Q}) \mathrm{d} t \\
& \equiv \delta \int_{0}^{T} \frac{1}{2} M \dot{Z}^{2}+\frac{1}{2} L_{i} \dot{Q}^{2}-M g Z-\frac{1}{2} k_{s} Z^{2}-K(Z) \dot{Q} \mathrm{~d} t
\end{aligned}
$$

with $K(Z) \equiv \int^{Z} \gamma G(\hat{Z}) \mathrm{d} \hat{Z}$.

A Legendre transform of (A.20) yields the following conjugate momenta

$$
P_{Q} \equiv \frac{\partial \mathrm{L}_{m m}}{\partial \dot{Q}}=L_{i} \dot{Q}-K(Z) \quad \text { and } \quad M W \equiv \frac{\partial \mathrm{L}_{m m}}{\partial \dot{Z}}=M \dot{Z}
$$

The Hamiltonian $H\left(Z, W, Q, P_{Q}\right)$ is then the Legendre transform of $\mathrm{L}(Q, \dot{Q}, Z, \dot{Z})$ as follows

$$
\begin{aligned}
H\left(Z, W, Q, P_{Q}\right) & =P_{Q} \dot{Q}+M W \dot{Z}-\mathrm{L}_{m m}(Q, \dot{Q}, Z, \dot{Z}) \\
& =\frac{1}{2} \frac{\left(P_{Q}+K(Z)\right)^{2}}{L_{i}}+\frac{1}{2} M W^{2}+M g Z+\frac{1}{2} k_{s} Z^{2} .
\end{aligned}
$$

The variational principle (A.20) in terms of these new variables then becomes

$$
\begin{aligned}
0= & \delta \int_{0}^{T} P_{Q} \dot{Q}+M W \dot{Z}-H\left(Z, W, Q, P_{Q}\right) \mathrm{d} t \\
= & \delta \int_{0}^{T} P_{Q} \dot{Q}+M W \dot{Z}-\frac{1}{2} \frac{\left(P_{Q}+K(Z)\right)^{2}}{L_{i}}-M g Z-\frac{1}{2}\left(M W^{2}+k_{s} Z^{2}\right) \mathrm{d} t \\
= & \int_{0}^{T}\left(\dot{Q}-\frac{\left(P_{Q}+K(Z)\right)}{L_{i}}\right) \Delta P+(M \dot{Z}-M W) \delta W \\
& \quad-\left(M \dot{W}+M g+k_{s} Z+K^{\prime}(Z) \frac{\left(P_{Q}+K(Z)\right)}{L_{i}}\right) \delta Z-\dot{P}_{Q} \delta Q \mathrm{~d} t
\end{aligned}
$$

yielding, cf. (A.18), the system of equations

$$
\begin{aligned}
\delta P_{Q}: & \dot{Q}=\frac{\left(P_{Q}+K(Z)\right)}{L_{i}} \equiv I, \quad \delta W: \quad M \dot{Z}=M W \\
\delta Q: & \dot{P}_{Q}=0, \quad \delta Z: \quad M \dot{W}+M g+k_{s} Z+K^{\prime}(Z) \frac{\left(P_{Q}+K(Z)\right)}{L_{i}}=0 .
\end{aligned}
$$

The modification (16) to the variational principle (A.23) without the potential energy of the spring and, instead, including the hydrodynamics was used in the main text. 
Table A1. Indicative parameter values and units used in three-dimensional numerical calculations for the wave-buoy system. LED: https://en.wikipedia.org/wiki/ Shockley_diode_equation.

\begin{tabular}{lll} 
Constant & value/unit & determination \\
\hline Magnetic dipole moment & $m=0.1 \mathrm{Am}^{2}$ & estimate \\
& $\mu_{0}=4 \pi 10^{-7} \mathrm{~N} / \mathrm{A}^{2}$ & \\
Coil outer radius & $a=0.04 \mathrm{~m}$ & estimate \\
& $\alpha_{h}=0.05,0.2$ & estimate \\
& $K=0.53$ & \\
Radius magnet & $A_{m}=0.032 \mathrm{~m}$ & estimate \\
Coil diameter & $D=0.2769 \mathrm{~mm}$ & coil MW30-9 \\
Length coil & $L=0.08 \mathrm{~m}$ & estimate \\
Length magnet & $L_{m}=0.04 \mathrm{~m}$ & estimate \\
Winding number & $N=L / D$ & calculated \\
Coil induction & $L_{i}=\mu_{0}\left(N^{2} / L\right) \pi a^{2}$ & calculated, see text \\
Coil resistance & $R_{c}=20.23 \mathrm{~V} / \mathrm{A}$ & calculated, see text \\
Circuit resistance & $R_{i}=R_{c}$ & estimate \\
Shockley & $n_{q}=1$ & www \\
Shockley voltage & $V_{T}=2.05 \mathrm{~V}$ & www \\
Shockley current & $I_{s a t}=0.02 \mathrm{~A}$ & www \\
Mast length & $H_{m}=0.2 \mathrm{~m}$ & estimate \\
Conductivity & $\sigma=5.96 \times 10^{7} \mathrm{~S} / \mathrm{m}$ & copper $/$ wiki \\
Mass & $M=0.08 \mathrm{~kg}$ & estimate \\
Keel & $H_{k}=0.04 \mathrm{~m}$ & estimate \\
\hline
\end{tabular}

